\title{
Progenitor mass constraints for the type Ib intermediate-luminosity SN 2015ap and the highly extinguished SN 2016bau
}

\author{
Amar Aryan $^{\star 1,2}$, S. B. Pandey ${ }^{1}$, WeiKang Zheng ${ }^{3}$, Alexei V. Filippenko ${ }^{3,4}$, \\ Jozsef Vinko ${ }^{5,6,7,8}$, Ryoma Ouchi ${ }^{9}$, Isaac Shivvers ${ }^{3}$, Heechan Yuk ${ }^{3,10}$, Sahana Kumar ${ }^{3,11}$, \\ Samantha Stegman ${ }^{3}$, Goni Halevi ${ }^{3,12}$, Timothy W. Ross ${ }^{3}$, Carolina Gould ${ }^{3}$, \\ Sameen Yunus ${ }^{3}$, Raphael Baer-Way ${ }^{3}$, Asia deGraw ${ }^{3}$, Keiichi Maeda ${ }^{9}$, \\ D. Bhattacharya ${ }^{13}$, Amit Kumar ${ }^{1,14}$, Rahul Gupta ${ }^{1,2}$, Abhay P. Yadav ${ }^{15}$,
} David A. H. Buckley ${ }^{16}$, Kuntal Misra ${ }^{1}$, and S. N. Tiwari ${ }^{2}$

${ }^{1}$ Aryabhatta Research Institute of Observational Sciences, Manora Peak, Nainital 263002 India

2 Department of Physics, Deen Dayal Upadhyay Gorakhpur University, Gorakhpur, Civil Lines, Gorakhpur (U.P.) 273 009, India

${ }^{3}$ Department of Astronomy, University of California, Berkeley, CA 94720-3411, USA

${ }^{4}$ Miller Institute for Basic Research in Science, University of California, Berkeley, CA 94720, USA

${ }^{5}$ Department of Astronomy, University of Texas at Austin, Austin, TX, USA

${ }^{6}$ CSFK Konkoly Observatory, Konkoly Thege M. ut 15-17, Budapest, 1121, Hungary

${ }^{7}$ Department of Optics and Quantum Electronics, University of Szeged, Dóm tér 9, Szeged, 6720 Hungary

${ }^{8}$ ELTE Eötvös Loránd University, Institute of Physics, Pázmány Péter sétány 1/A, Budapest, 1117 Hungary

${ }^{9}$ Department of Astronomy, Kyoto University, Kitashirakawa-Oiwake-cho, Sakyo-ku, Kyoto 606-8502, Japan

${ }^{10}$ Department of Physics and Astronomy, University of Oklahoma, 440 W. Brooks St., Norman, OK 73019, USA

${ }^{11}$ Department of Physics, Florida State University, Tallahassee, FL 32306, USA

${ }^{12}$ Department of Astrophysical Sciences, Princeton University, 4 Ivy Lane, Princeton, NJ 08540, USA

${ }^{13}$ The Inter-University Centre for Astronomy and Astrophysics (IUCAA), Ganeshkhind, Savitribai Phule Pune University Campus, Pune 411 007, India

${ }^{14}$ School of Studies in Physics and Astrophysics, Pt. Ravishankar Shukla University, Chattisgarh 492 010, India

${ }^{15}$ Department of Physics and Astronomy, National Institute of Technology Rourkela-769008, Odisha, India

${ }^{16}$ South African Astronomical Observatory, PO Box 9, Observatory 7935, Cape Town, South Africa

Accepted 2021 May 11. Received 2021 May 11; in original form 2020 December 24

\begin{abstract}
Photometric and spectroscopic analyses of the intermediate-luminosity Type Ib supernova (SN) 2015ap and of the heavily reddened Type Ib SN 2016bau are discussed. Photometric properties of the two SNe, such as colour evolution, bolometric luminosity, photospheric radius, temperature, and velocity evolution, are also constrained. The ejecta mass, synthesised nickel mass, and kinetic energy of the ejecta are calculated from their light-curve analysis. We also model and compare the spectra of SN 2015ap and SN 2016bau at various stages of their evolution. The P Cygni profiles of various lines present in the spectra are used to determine the velocity evolution of the ejecta. To account for the observed photometric and spectroscopic properties of the two SNe, we have computed $12 M_{\odot}$ zero-age main sequence (ZAMS) star models and evolved them until the onset of core collapse using the publicly available stellarevolution code MESA. Synthetic explosions were produced using the public version of STELLA and another publicly available code, SNEC, utilising the MESA models. SNEC and STELLA provide various observable properties such as the bolometric luminosity and velocity evolution. The parameters produced by SNEC/STELLA and our observations show close agreement with each other, thus supporting a $12 M_{\odot}$ ZAMS star as the possible progenitor for SN 2015ap, while the progenitor of SN 2016bau is slightly less massive, being close to the boundary between SN and non-SN as the final product.
\end{abstract}

Key words: supernovae: general - supernovae: individual: SN 2016bau, SN 2015ap techniques: photometric - techniques: spectroscopic 


\section{INTRODUCTION}

Core-collapse supernovae (CCSNe) are among the most powerful astronomical explosions, occurring during the final stellar evolutionary stages of massive stars $\left(M>8-10 M_{\odot}\right.$; e.g., Garry 2004; Woosley 2005; Groh 2017). Extensive reviews of various types of CCSNe and criteria used to categorise them are provided by (among others) Filippenko (1997) and GalYam (2016). CCSNe are broadly classified according to the presence or absence of hydrogen $(\mathrm{H})$ features in their spectra. SNe showing prominent $\mathrm{H}$ features are classified as Type II, while those lacking them are Type I. These classes are further divided into various subclasses. Type Ib SNe exhibit prominent helium (He) features in their spectra, whereas Type Ic SNe show neither H nor He obvious features. Prominent features of intermediate-mass elements such as $\mathrm{O}, \mathrm{Mg}$, and $\mathrm{Ca}$ are also seen in Type Ib and Type Ic SN spectra. Although $\mathrm{SNe} \mathrm{Ib/c} \mathrm{lack} \mathrm{obvious} \mathrm{H}$ features in their early-time spectra, few studies have focused on the existence of $\mathrm{H}$ in SNe Ib (e.g., Branch et al. 2002, 2006; Hachinger et al. 2012; Elmhamdi et al. 2006). Type IIb SNe form a transition class of objects that link SNe II and SNe Ib (Filippenko 1988; Filippenko et al. 1993; Smartt 2009). The early-phase spectra of SNe IIb display prominent $\mathrm{H}$ features, while unambiguous He features appear after a few weeks.

The main powering mechanism in normal $\mathrm{SNe} \mathrm{Ib} / \mathrm{c}$ is radioactive decay of ${ }^{56} \mathrm{Ni}$ and ${ }^{56} \mathrm{Co}$, leading to the deposition of energetic gamma rays that thermalise in the homologously expanding ejecta (e.g., Arnett 1980, 1982, 1996; Nadyozhin 1994; Chatzopoulous et al. 2013; Nicholl et al. 2017). Some SNe Ib (e.g., SN2005bf, Maeda et al. 2007) have also shown evidence for the light curves being powered by the spindown of a young magnetar (e.g., Ostriker \& Gunn 1971; Arnett \& Fu 1989; Maeda et al. 2007; Kasen \& Bildsten 2010; Woosley 2010; Chatzopoulous et al. 2013; Nicholl et al. 2017). In the post-photospheric phase, when the SN ejecta become optically thin, the light curves of CCSNe are powered by energy deposition from the radioactive decay of ${ }^{56} \mathrm{Ni}$ to ${ }^{56} \mathrm{Co}$ and finally to ${ }^{56} \mathrm{Fe}$. In many cases, however, the SN progenitor is embedded within dense circumstellar matter (CSM), so when the SN explosion occurs the SN ejecta may violently interact with the CSM, resulting in the formation of forward and reverse shocks that deposit their kinetic energy into the material which is radiatively released and thus powers the light curve (e.g., Chevalier \& Fransson 1982, 1994; Moriya et al. 2011; Ginzberg \& Balberg 2012; Chatzopoulous et al. 2013; Nicholl et al. 2017).

Understanding the possible progenitors of H-stripped CCSNe is still a challenging task, though a few studies have been performed. For SNe Ib/c, broadly two scenarios are proposed. The first involves relatively low-mass progenitors $\left(>11 M_{\odot}\right)$ in binary systems (Podsiadlowski et al. 1992; Nomoto et al. 1995; Smartt 2009), where the primary star lost its $\mathrm{H}$ envelope through transfer of mass to a companion star. The second considers massive Wolf-Rayet (WR) stars (> 20-25 $M_{\odot}$ ) that lose mass via stellar winds (e.g., Gaskell et al. 1986; Eldridge et al. 2011; Groh et al. 2013). In one relatively recent study, Cao et al. (2013) reported a possible progenitor of SN iPTF13bvn identified in pre-explosion images within a $2 \sigma$ error radius, consistent with a massive WR progenitor star. The massive WR progenitor scenario is also supported by stellar evolutionary models. Based on observa- tional evidence, from early- and nebular-phase spectroscopy of SNe Ib, both massive WR stars as well as interacting binary progenitors are proposed. For $\mathrm{SNe} \mathrm{IIb}$, the direct detections of objects in pre-explosion images of four cases also indicate either massive WR stars $\left(M_{\mathrm{ZAMS}} \approx 10-28 M_{\odot}\right.$; Crockett et al. 2008) or more extended yellow supergiants (YSGs) with $M_{\mathrm{ZAMS}}=12-17 M_{\odot}$ (Van Dyk et al. 2013 ; Folatelli et al. 2014; Smartt 2015) as possible progenitors. The hydrodynamical modelling of the possible progenitors (identified either via direct imaging as in the case of iptf13bvn (Cao et al. 2013) or indirect methods which include nebularphase spectral modelling (Jerkstrand 2015; Uomoto 1986)) and simulating their synthetic explosions can be vital to understanding their nature, physical conditions, circumstellar environment, and chemical compositions. Unfortunately, only a handful of such studies have been performed in the cases of stripped-envelope $\mathrm{SNe}$, including the Type Ib SN iptf13bvn (Cao et al. 2013; Bersten et al. 2014; Paxton et al. 2018), the famous Type IIb SN 2016gkg (Bersten et al. 2018), and the Type IIb SN 2011dh (Bersten et al. 2012). Our work takes such studies one step further as we perform hydrodynamical simulations of the possible progenitors of two $\mathrm{SNe} \mathrm{Ib}$ and also simulate their synthetic explosions.

In this paper, we explore the photometric and spectroscopic behaviour of SN 2015ap and SN 2016bau, primarily using data obtained using the KAIT, Nickel, and Shane telescopes at Lick Observatory. Based on the analysis, we model and attempt to place constraints on the properties of the progenitors of these two SNe. In Sec. 2, details about various telescopes and reduction procedures are presented. Sec. 3 provides methods to correct for the Milky Way and the hostgalaxy extinction. Photometric properties of the two SNe, such as their bolometric light curve, temperature, radius, and velocity evolution, are also discussed. Sec. 4 includes the analysis describing the spectral evolution of SN 2015ap and SN 2016bau, as well as comparisons with other similar and well-studied SNe; we also model the spectra of these $\mathrm{SNe}$ using SYN++. Quasi-bolometric light-curve modelling is performed and discussed in Sec. 5; light curves corresponding to various powering mechanisms of $\mathrm{SNe}$ are fitted to the observed quasi-bolometric light curves. The assumptions and methods for modelling the possible progenitors of the two $\mathrm{SNe}$ and their evolution until the onset of core-collapse using MESA are presented in Sec. 6. We discuss the assumptions and methods for producing the synthetic explosions using SNEC and STELLA in Sec. 7; here, the comparisons between the parameters obtained through synthetic explosions and observed ones are presented. In Sec. 8, we discuss the major results and findings of our analysis. Finally, in Sec. 9, we summarise our work by briefly discussing the major outcomes and provide concluding remarks.

\section{DATA ACQUISITION AND REDUCTION}

SN 2015ap was discovered by KAIT as part of the Lick Observatory Supernova Search (LOSS; Filippenko et al. 2001), in an $18 \mathrm{~s}$ unfiltered image (close to the $R$ band; see Li et al. 2003) taken at 11:16:31 on 2015 Sep. 08 (Ross et al. 2015), at $17.41 \pm 0.06 \mathrm{mag}$. The object was also marginally detected one day earlier on Sep. 07.47 with $18.07 \pm 0.22$ mag. We measure its J2000.0 coordinates to be $\alpha=02^{\mathrm{h}} 05^{\mathrm{m}} 13.32^{\mathrm{s}}$, 

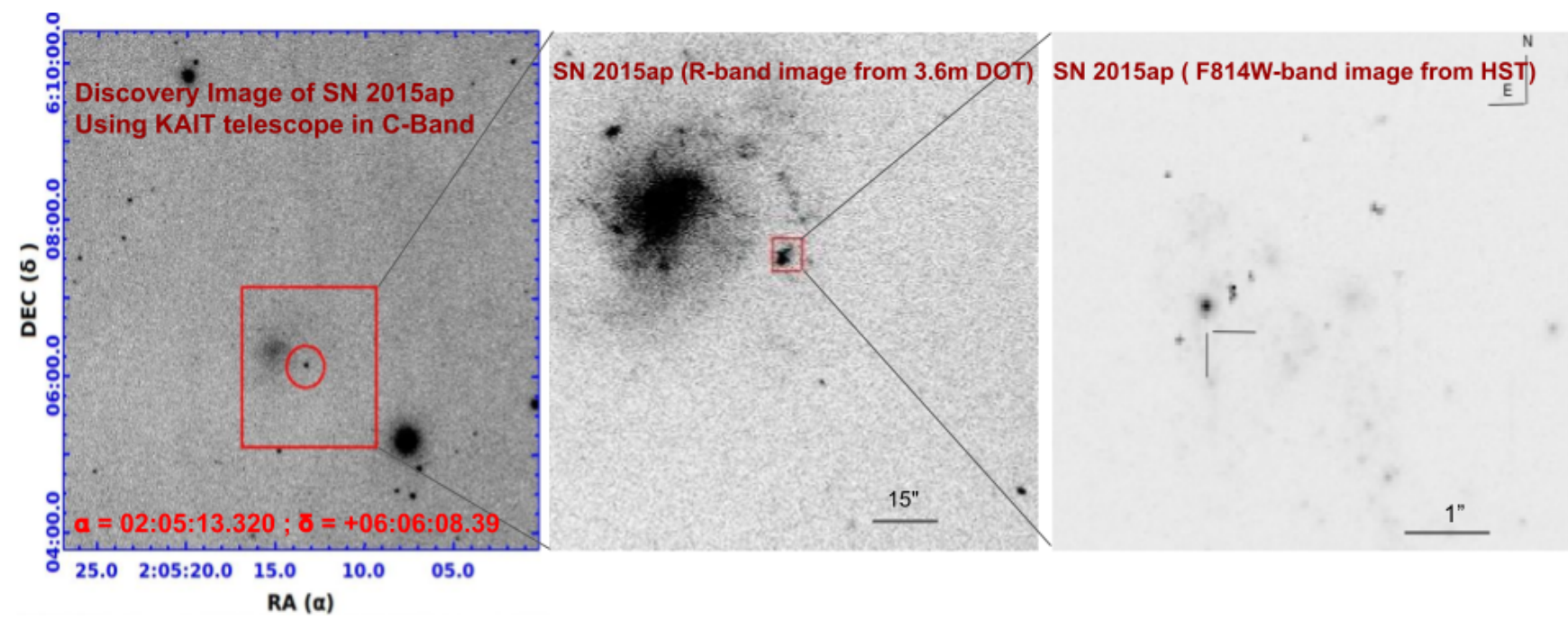

Figure 1. Left panel: The discovery image of SN 2015ap using the KAIT telescope in C-filter.The SN is marked inside the red circle. . Middle panel: A zoomed version of an $R$-band image with $2^{\prime} \times 2^{\prime}$ FoV obtained on 6 Oct. 2020 using a $4 \mathrm{k} \times 4 \mathrm{k}$ CCD imager mounted on the $3.6 \mathrm{~m}$ DOT. Right panel: A further zoomed, about $6^{\prime \prime} \times 6^{\prime \prime}$ FoV of the location of SN 2015ap taken on 12 Aug. 2020 using the Hubble Space Telescope (HST) in the F $814 \mathrm{~W}$ filter. The SN seems to be fainter than the detection limit of HST at this time. A log of $H S T$ observations is included in the Appendix.

$\delta=+06^{\circ} 06^{\prime} 08^{\prime \prime} .4$, with an uncertainty of $0^{\prime \prime} 5$ in each coordinate. SN 2015ap is $28^{\prime \prime} \cdot 3$ west and $166^{\prime \prime} 2$ south of the nucleus of its host galaxy IC 1776, which has a redshift of $z=0.011375 \pm 0.000017$ (Chengalur et al. 1993) and a barred-spiral morphology (SB(s)d; de Vaucouleurs et al. 1991)). SN 2016bau was discovered by Ron Arbour in an unfiltered image taken at 23:22:33 on 2016 Mar. 13 (Arbour 2016), at $17.8 \mathrm{mag}$. Its J2000.0 coordinates are given as $\alpha=11^{\mathrm{h}} 20^{\mathrm{m}} 59.02 \mathrm{~s}, \delta=+53^{\circ} 10^{\prime} 25^{\prime \prime}$. 6 . SN 2016bau is $35^{\prime \prime} 3$ west and $15^{\prime \prime} .2$ north of the nucleus of its host galaxy NGC 3631, which has $z=0.00384 \pm 0.00014$ (Falco et al. 1999) and a morphology of SAc C (Ann, Seo \& Ha 2015). Both of these $\mathrm{SNe}$ were classified as $\mathrm{SN} \mathrm{Ib}$ on the basis of well-developed features of He I, Fe II (blended), and Ca II, a few days after maximum brightness.

Figure 1 shows finder charts of SN 2015ap obtained with different telescopes. The $B, V, R$, and $I$ follow-up images of SN 2015ap and SN 2016bau were obtained with both the $0.76 \mathrm{~m}$ Katzman Automatic Imaging Telescope (KAIT; Filippenko et al. 2001) and the $1 \mathrm{~m}$ Nickel telescope at Lick Observatory. All images were reduced using a custom pipeline ${ }^{1}$ detailed by Stahl et al. (2019). Here, we briefly summarise the process for photometry. The image-subtraction procedures were applied in order to remove the host-galaxy light, using additional images obtained after the SN had faded below our detection limit. Point-spread-function (PSF) photometry was obtained using DAOPHOT (Stetson 1987) from the IDL Astronomy User's Library ${ }^{2}$. Three nearby stars were chosen from the Pan-STARRS1 ${ }^{3}$ catalogue for calibration. Their magnitudes were first transformed into Landolt (1992) magnitudes using the empirical prescription presented by Torny et al. (2012, Eq. 6) and then transformed to the
KAIT/Nickel natural system. Apparent magnitudes were all measured in the KAIT4/Nickel2 natural system. The final results were transformed to the standard system using local calibrators and colour terms for KAIT4 and Nickel2 (Stahl et al. 2019). In addition, the $U$-band photometry was obtained from the Swift Optical/Ultraviolet Supernova Archive (SOUSA; https://archive.stsci.edu/prepds/sousa/; Brown et al. 2014).

There are a total of 17 spectra of SN 2015ap; 15 of them were taken with the Kast spectrograph ${ }^{4}$ (Miler \& Stone 1993) on the $3 \mathrm{~m}$ Shane telescope at Lick Observatory and the other 2 were taken with $\operatorname{LRIS}^{5}$ (Oke et al. 1995) on the Keck-I $10 \mathrm{~m}$ telescope. Except for the earliest spectrum taken on 10.531 Sep. 2015 (UT dates are used throughout this paper) using the Lick Shane/Kast system, 16 spectra in this paper were published by Shivvers et al. (2018); refer to that paper for details of the observations. Also, there are 8 spectra of SN 2016bau, all obtained with the Lick Shane/Kast system. We used the 600/4310 grism on the blue side, the $300 / 7500$ grating on the red side, the d5500 dichroic, and a long slit $2^{\prime \prime}$ wide. For LRIS we used the $600 / 4000$ grism on the blue side, the 400/8500 grating on the red side, and a $1^{\prime \prime}$ slit. The spectra were binned to $2 \AA$ pixel $^{-1}$.

\section{PHOTOMETRIC PROPERTIES}

In this section, we discuss various photometric properties of SN 2015ap and SN 2016bau, including their colour evolution, extinction, quasi-bolometric light curves, and various blackbody parameters.

\footnotetext{
4 https://mthamilton.ucolick.org/techdocs/instruments/kast

5 https://www2.keck.hawaii.edu/inst/lris/lrishome.html
}

\footnotetext{
1 https://github.com/benstahl92/LOSSPhotPypeline

2 http://idlastro.gsfc.nasa.gov/

3 http://archive.stsci.edu/panstarrs/search.php
} 


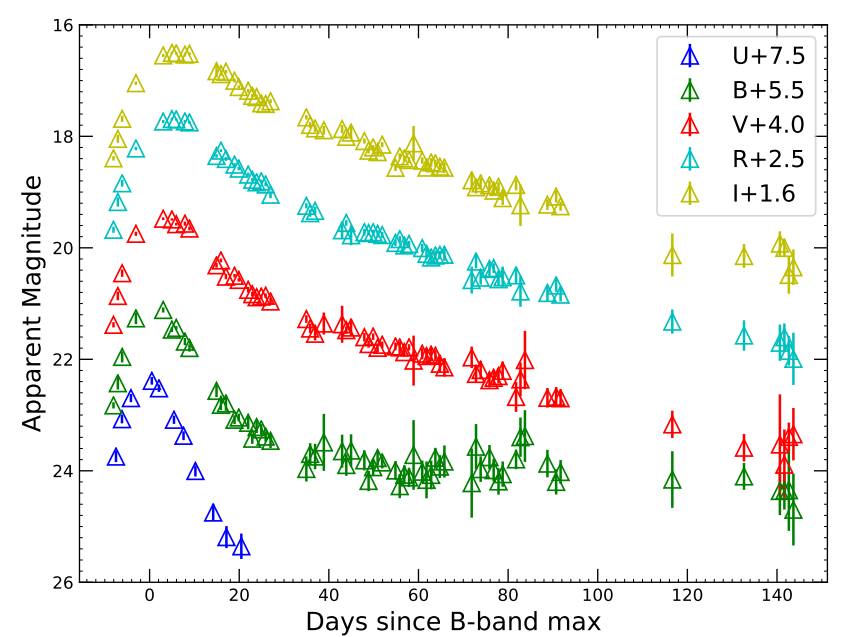

Figure 2. $U B V R I$ light curves of SN 2015ap, where $B V R I$ data were obtained with KAIT while the $U$-band data were taken from the UVOT mounted on Swift (https://swift.gsfc.nasa.gov).

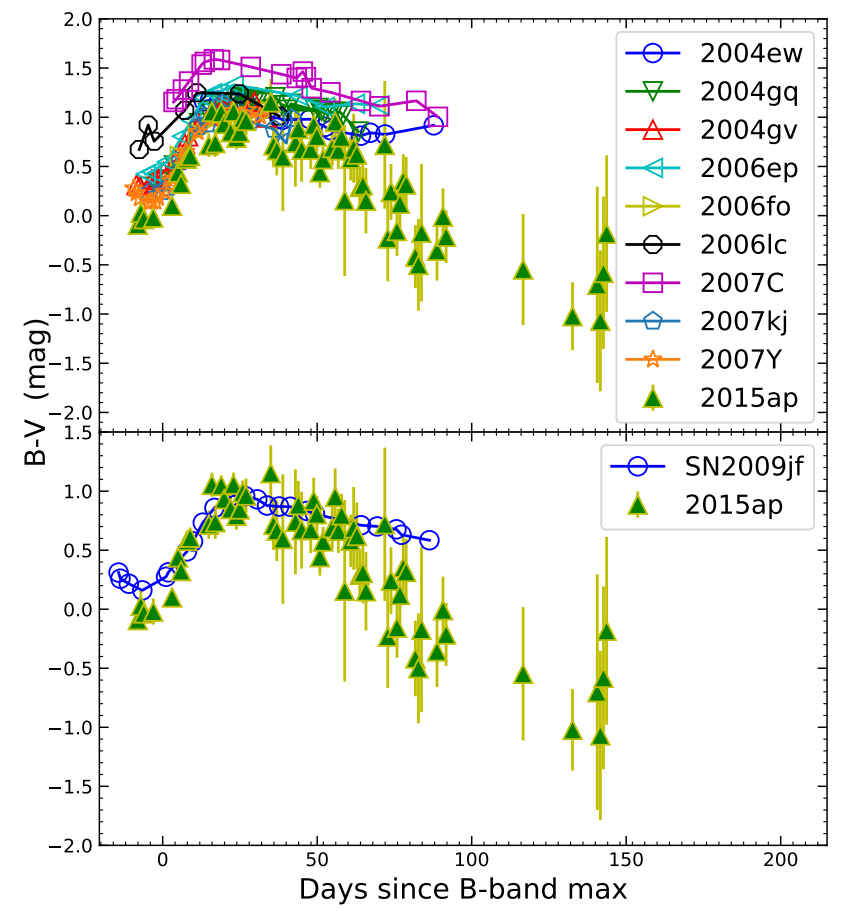

Figure 3. The top panel shows a comparison of the $(B-V)$ colour of SN 2015ap (corrected for Milky Way extinction) with that of other Type Ib SNe. The data for SNe other than SN 2015ap are taken from Stritzinger et al. (2018). The bottom panel shows the $(B-V)$ colour curves of SN 2015ap and SN 2009jf (both corrected for Milky Way extinction).

\subsection{Photometric properties of SN2015ap}

Most of the analysis in this paper has been performed with respect to $B$-band maximum brightness. To find its date, we fit a sixth-order polynomial to the $B$ data which well sample the photospheric phase. The resulting date of $B$-band maximum is MJD $57282.47 \pm 2.56$. To determine the explosion epoch $\left(t_{\exp }\right)$ of SN 2015ap, we use $R$-band data. The $R$ magnitudes are converted into fluxes and a sixth-order polynomial is fitted. We extrapolate the polynomial and the epoch corresponding to zero flux is taken as the explosion epoch, MJD 57272.72 \pm 1.49 , which is in good agreement with Prentice et al. (2019).

Figure 2 shows the $U B V R I$ light curves of SN 2015ap. The rise rate for the $U$-band light curve is faster than that of other bands, and similarly the $U$ decline rate is faster, making the $U$ light curve much narrower compared to other bands. As we go to longer wavelengths, we see that the light curves become broader, with the $I$-band light curve being the broadest.

\subsubsection{Colour evolution and extinction correction}

Distances are taken from the NASA Extragalctic Database $^{6}$ (NED), and a cosmological model with $\mathrm{H}_{0}=73.8 \mathrm{~km} \mathrm{~s}^{-1} \mathrm{Mpc}^{-1}, \Omega_{m}=0.3$, and $\Omega_{\Lambda}=0.7$ is assumed throughout. For SN 2015ap, we corrected for Milky Way (MW) extinction using NED following Schlafly \& Finkbeiner (2011). In the direction of SN 2015ap, the Galactic extinctions for the $U, B, V, R$, and $I$ bands are $0.185,0.154,0.117,0.092$, and $0.064 \mathrm{mag}$, respectively.

The top panel of Figure 3 shows a comparison of the $(B-V)$ colour of SN 2015ap with that of other Type Ib SNe (all corrected for MW extinction). SN 2015ap seems to be the least reddened and lies below nearly all of the other $\mathrm{SNe} \mathrm{Ib}$.

Following Prentice et al. (2019), the host-galaxy contamination is negligible and hence ignored. To further support this assumption, the $(B-V)$ colour curve of SN 2009jf (Sahu et al. 2011) is shown in the bottom panel of Figure 3 , corrected for an MW colour excess of $E(B-V)_{\mathrm{MW}}=$ $0.112 \mathrm{mag}$ (host extinction is negligible). In order to match the $(B-V)$ colour curve of SN 2009jf, we need not apply any shift to the MW-corrected SN 2015ap $(B-V)$ colour curve.

Figure 4 illustrates the position of SN 2015ap in the Miller diagram (Richardson et al. 2014). The distance modulus $(\mu)$ of SN 2015ap is 33.269 mag; thus, SN 2015ap appears to be a normal SN Ib. For SN 2016bau, we calculate $\mu=32.65$ mag; based on its position in Figure 4, it seems to be a moderately luminous, normal SN Ib.

\subsubsection{Quasi-bolometric and bolometric light curves}

To obtain the quasi-bolometric light curves, we made use of the superbol code (Nicholl M. 2018). We first provided the extinction-corrected $U, B, V, R$, and $I$ data to superbol. Thereafter, it mapped the light curve in each filter to a common set of times through the processes of interpolation and extrapolation. It then fit blackbodies to the spectral energy distribution (SED) at each epoch, up to the observed wavelength, to give the quasi-bolometric light curve by performing trapezoidal integration. The peak quasi-bolometric luminosity obtained through integrating the flux over a wave-

6 https://ned.ipac.caltech.edu/ 


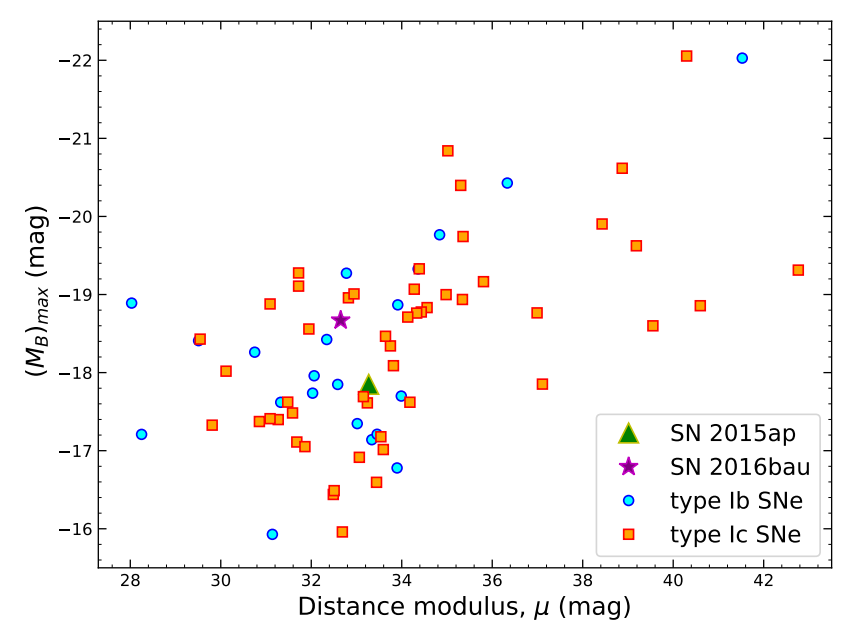

Figure 4. The position of SN 2015ap and SN 2016bau in the Miller diagram.

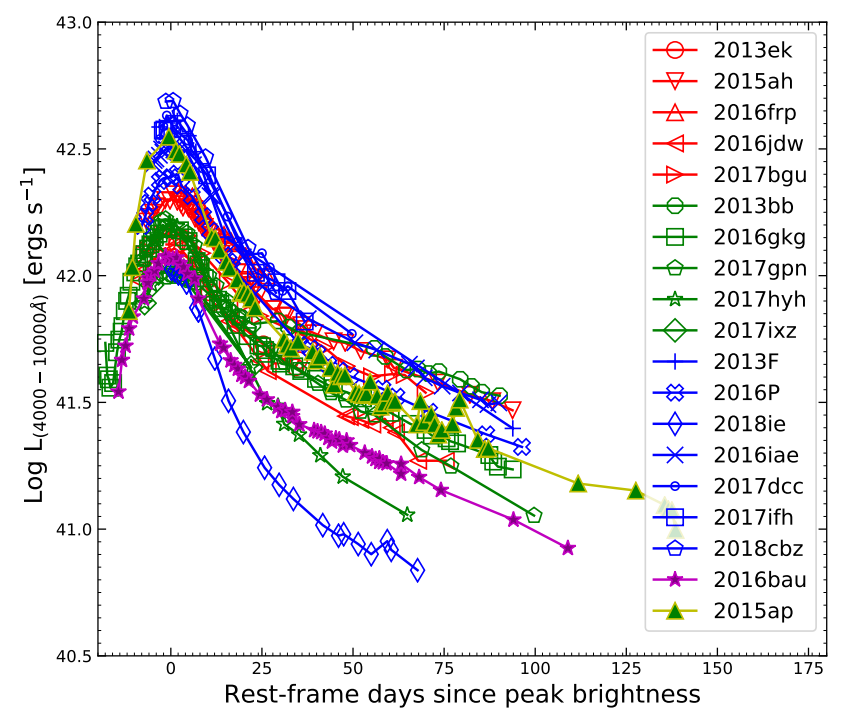

Figure 5. Comparison of quasi-bolometric light curves of SN 2015ap and SN 2016bau, obtained by fitting a blackbody to the SED and integrating the fluxes over the wavelength range of $4000-10,000 \AA$, with that of other stripped-envelope CCSNe. Symbols are colour coded: red, green, and blue correspond to Type Ib, IIb, and Ic SNe, respectively.

length range of $4000-10,000 \AA$ is $10^{(42.548 \pm 0.019)} \mathrm{erg} \mathrm{s}^{-1}$, in agreement with Prentice et al. (2019).

Figure 5 shows a comparison of the quasi-bolometric light curve of SN 2015ap with that of other H-stripped CCSNe. The code superbol also provides the bolometric light curve, including the additional blackbody corrections to the observed quasi-bolometric light curve, by fitting a single blackbody to observed fluxes at a particular epoch and integrating the fluxes trapezoidally for a wavelength range of $100-25,000 \AA$. The top panel of Figure 6 provides the resulting quasi-bolometric and bolometric light curves of SN 2015ap.

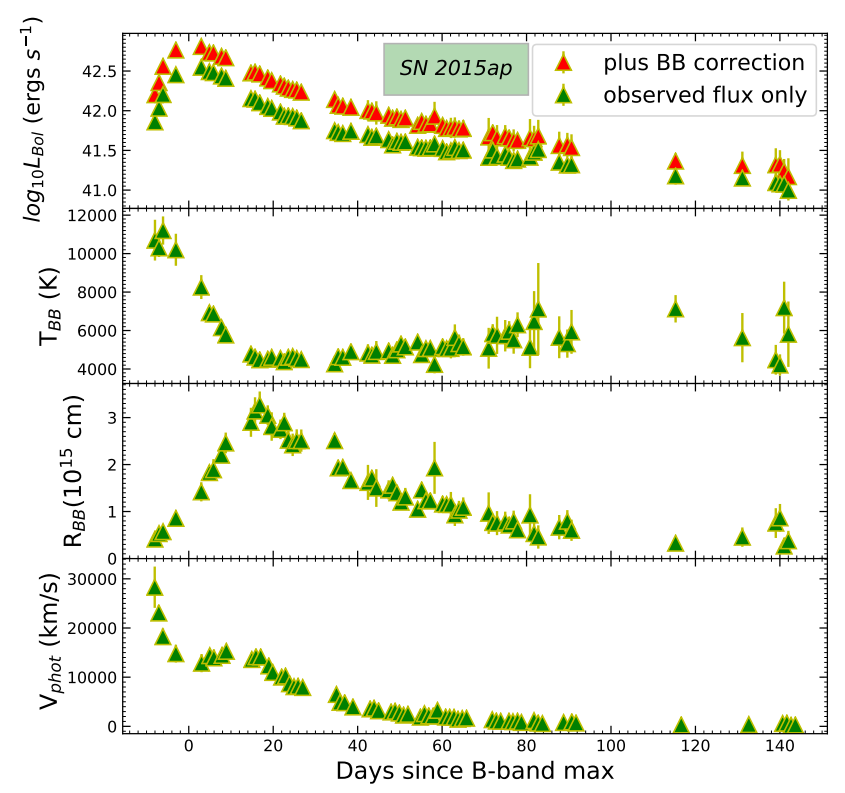

Figure 6. The top panel shows the bolometric and quasibolometric light curves of SN 2015ap. Second panel: the temperature evolution of SN 2015ap. Third and fourth panels: the radius and velocity evolutions (respectively) obtained using blackbody fits.

\subsubsection{Temperature, radius, and velocity evolution}

From superbol, the photospheric temperature $\left(T_{\mathrm{BB}}\right)$ and radius $\left(R_{\mathrm{BB}}\right)$ evolution of SN 2015ap are also obtained. During the initial phases, the photospheric temperature is high, reaching about $11,400 \mathrm{~K}$ at $-6.18 \mathrm{~d}$. Further, as the SN ejecta expand, cooling occurs and the temperature tends to fall, dropping to $4490 \mathrm{~K}$ on $+21.66 \mathrm{~d}$, then remaining nearly constant (Fig. 6, second panel from top). A conventional evolution in radius is also seen. Initially, at an epoch of $-8 \mathrm{~d}$, the photospheric radius is $4.14 \times 10^{14} \mathrm{~cm}$. Thereafter, the SN expands and its radius increases, reaching a maximum radius of $3.27 \times 10^{15} \mathrm{~cm}$, beyond which the photosphere seems to recede into the SN ejecta (Fig. 6, third panel from top). From the prior knowledge of the explosion epoch and radii at various epochs, we can estimate the photospheric velocity evolution of this $\mathrm{SN}$, using $v_{\mathrm{ph}}=R_{\mathrm{BB}} / t$, where $t$ is the time since explosion. The bottom panel of Figure 6 shows the velocity evolution of SN 2015ap.

From the known value of $t_{\exp }$, a rise time $\left(t_{\text {rise }}\right)$ of $14.8 \pm 2.2 \mathrm{~d}$ is obtained. The photospheric velocity near maximum light is $9000 \mathrm{~km} \mathrm{~s}^{-1}$ (Prentice et al. 2019). With the known values of $t_{\text {rise }}$, the photospheric velocity near maximum light, and a constant opacity $(\kappa)$ of $0.07 \mathrm{~cm}^{2} \mathrm{~g}^{-1}$, we also obtain the ejecta mass $\left(M_{\mathrm{ej}}\right)$ and kinetic energy $\left(E_{\mathrm{ke}}\right)$ from the Arnett (1982) model by following Equations (1) and (3) of Wheeler et al. (2015): $2.2 \pm 0.6 M_{\odot}$ and $(1.05 \pm 0.31) \times 10^{51} \mathrm{erg}$, respectively. Our derived ejecta mass is slightly higher than that of Prentice et al. (2019), but less than Gangopadhyay et al. (2020). Corresponding to a peak luminosity of $(3.53 \pm 0.16) \times 10^{42} \mathrm{erg} \mathrm{s}^{-1}$, the amount of ${ }^{56} \mathrm{Ni}$ synthesised is $0.14 \pm 0.02 M_{\odot}$, calculated following Prentice et al. (2016). 


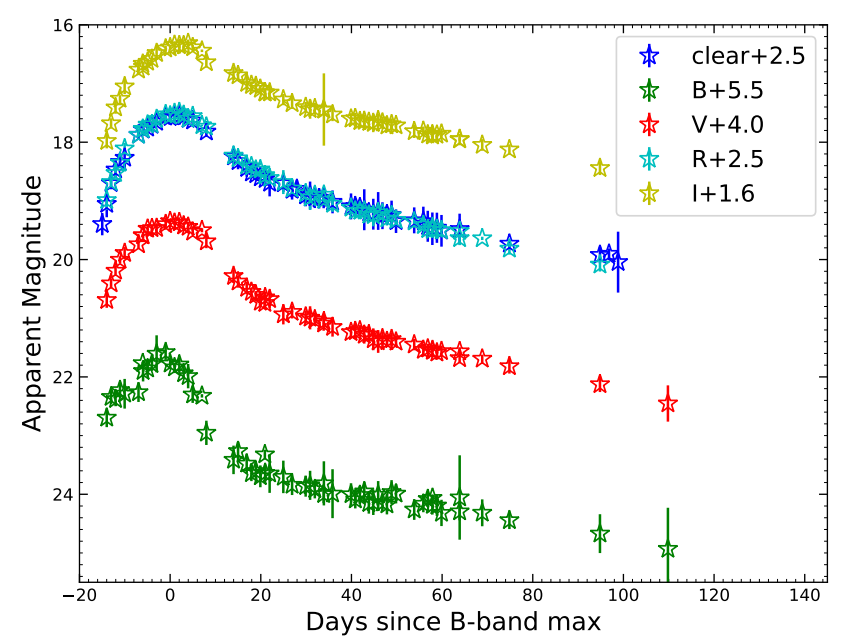

Figure 7. BVRI and Clear filter light curves of SN 2016bau.

\subsection{Photometric properties of SN 2016bau}

Following a method similar to that for SN 2015ap, the $B$ band maximum and the explosion epochs of SN 2016bau were determined to be MJD $57477.37 \pm 1.99$ and MJD $57462.54 \pm 0.97$, respectively. Figure 7 shows the $B V R I$ and Clear $(C)$ filter light curves of SN 2016bau. Light curves in the shorter-wavelength bands are narrower compared to those in the longer-wavelength bands, following a trend similar to that of SN 2015ap. Also, the $C$ filter almost exactly replicates the $R$-band light curve, as expected ( $\mathrm{Li}$ et al. 2003).

\subsubsection{Colour evolution and extinction correction}

Similar to SN 2015ap, we corrected for MW extinction using NED following Schlafly \& Finkbeiner (2011). In the direction of SN 2016bau, the Galactic extinction for the $B, V, R$, and $I$ bands is $0.060,0.045,0.036$, and $0.025 \mathrm{mag}$, respectively. The top panel of Figure 8 shows a comparison of the $(B-V)$ colour of SN 2016bau with that of other SNe Ib. SN 2016bau seems to be heavily reddened and lies above nearly all of the other SNe Ib. To correct for the host-galaxy extinction, we made use of the colour curve of SN 2009jf. We calculated the differences in the colours of the two SNe and took their weighted mean. In this calculation, we made use of data only in the range 0 to $+20 \mathrm{~d}$ for the reasons mentioned by Stritzinger et al. (2018). The resulting host-galaxy extinction is $E(B-V)_{\text {host }}=0.566 \pm 0.046$ mag. Thus, in order to match the SN 2009jf $(B-V)$ colour curve, we need to shift the MW-corrected $(B-V)$ colour curve of SN $2016 \mathrm{bau}$ downward by $0.566 \mathrm{mag}$, as shown in the bottom panel of Figure 8 .

\subsubsection{Quasi-bolometric and bolometric light curves}

To obtain the quasi-bolometric and bolometric light curves of SN 2016bau, we used superbol, as in the case of SN 2015ap. The extinction-corrected $B, V, R$, and $I$ data were given as input to superbol. The top panel of Figure 9 shows the quasi-bolometric and bolometric light curves of

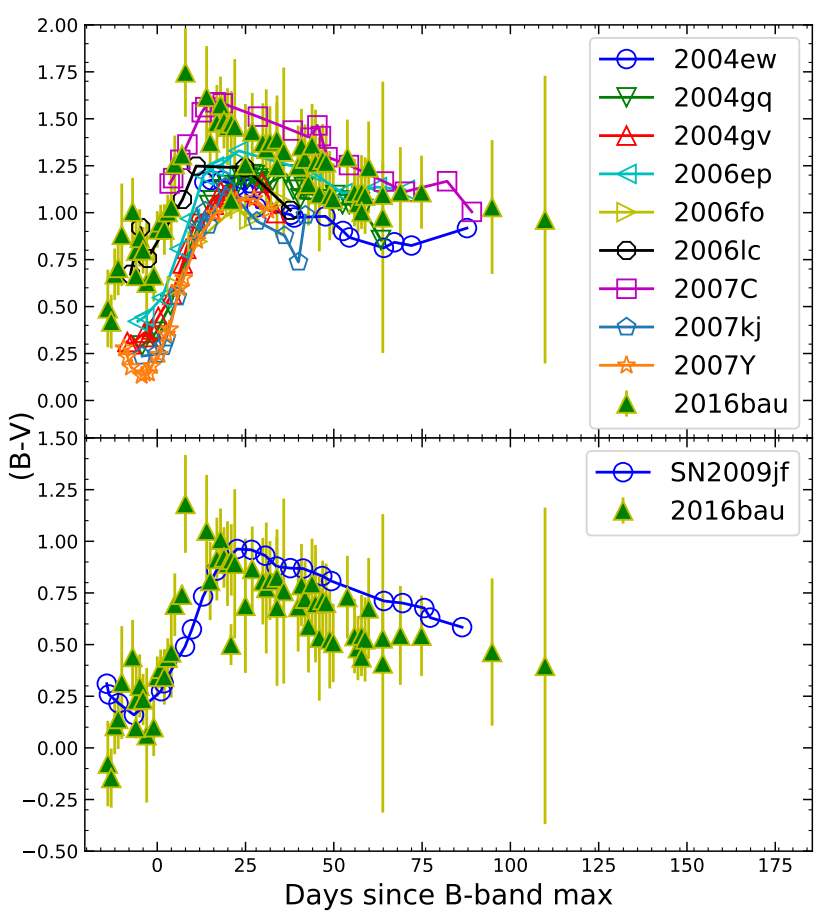

Figure 8. The top panel shows a comparison of the $(B-V)$ colour of SN 2016bau with that of other SNe Ib (all corrected for MW extinction). The data for the other SNe are taken from Stritzinger et al. (2018). The bottom panel shows the $(B-V)$ colour curves of SN 2016bau and SN 2009jf, both corrected for MW extinction. To match the SN 2009jf colour curve, a shift of 0.566 mag is required for the SN 2016bau colour curve.

SN 2016bau. Figure 5 shows the comparison of the quasibolometric light curve of SN 2016bau obtained by integrating the flux over the wavelength range 4000-10,000 $\AA$ with other H-stripped CCSNe from Prentice et al. (2019). Here, SN 2016bau also seems to lie on the moderately bright end.

\subsubsection{Temperature, radius, and velocity evolution}

Figure 9 also shows the evolution of the photospheric temperature $\left(T_{\mathrm{BB}}\right)$ and radius $\left(R_{\mathrm{BB}}\right)$ of $\mathrm{SN} 2016 \mathrm{bau}$, obtained using superbol. During the initial phases, the photospheric temperature is very high, reaching about $17,000 \mathrm{~K}$ near $0 \mathrm{~d}$. Thereafter, as the SN ejecta expand, cooling occurs and the temperature falls, reaching $6000 \mathrm{~K}$ at around $+20 \mathrm{~d}$, then remaining nearly constant (Fig. 9, second panel from top). A conventional evolution in radius is also seen (Fig. 9, third panel from top). Initially, at an epoch of around $-6 \mathrm{~d}$, the photospheric radius is $0.28 \times 10^{15} \mathrm{~cm}$. Following this, the supernova expands and its radius increases, reaching a maximum radius of $0.96 \times 10^{15} \mathrm{~cm}$, beyond which the photosphere seems to recede within the SN ejecta.

From the prior knowledge of explosion epoch and radii at various epochs, we estimate the photospheric velocity evolution of this SN in the same way as for SN 2015ap. The bottom panel of Figure 9 shows the velocity evolution of SN 2016bau.

From the prior knowledge of $t_{\mathrm{exp}}$, a rise time $\left(t_{\text {rise }}\right)$ of $17.09 \pm 1.29 \mathrm{~d}$ is obtained. The photospheric velocity near 


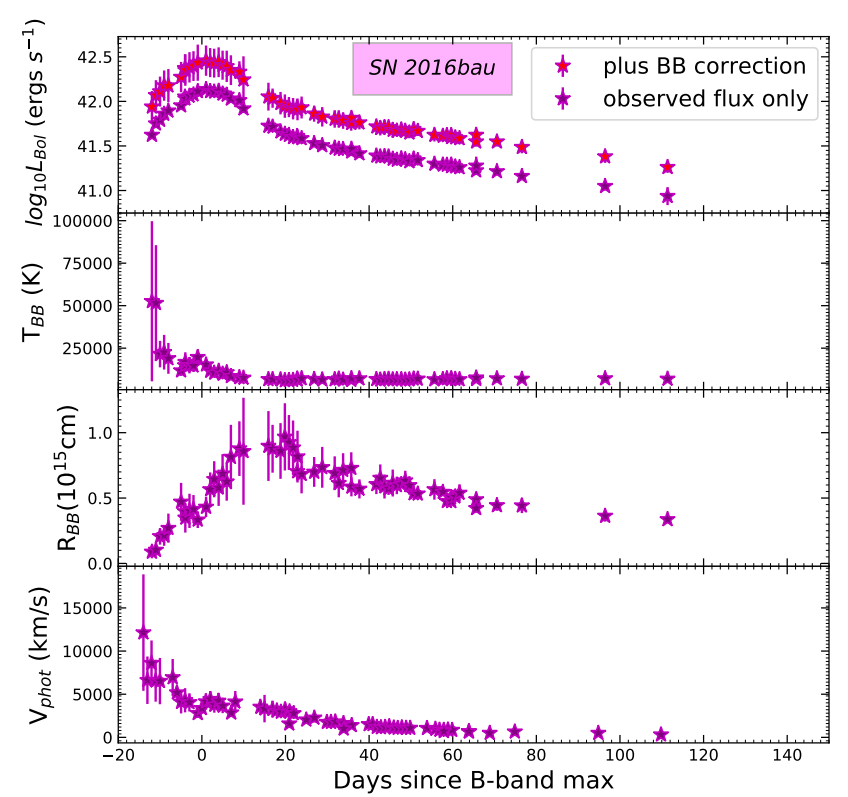

Figure 9. The top panel shows the bolometric and quasibolometric light curves of SN 2016bau. Second panel: the temperature evolution of SN 2016bau. Third and fourth panels: the radius and velocity evolution (respectively) obtained using blackbody fits.

maximum light is $\sim 5000 \mathrm{~km} \mathrm{~s}^{-1}$, obtained from a blackbody fit. With the known values of $t_{\text {rise }}$, the photospheric velocity near maximum light, and a constant opacity $(\kappa)$ $0.07 \mathrm{~cm}^{2} \mathrm{~g}^{-1}$, we obtain the ejecta mass $\left(M_{\mathrm{ej}}\right)$ and kinetic energy $\left(E_{\mathrm{ke}}\right)$ following Equation (1) and Equation (3) of Wheeler et al. (2015); the results are $1.6 \pm 0.3 M_{\odot}$ and $(0.24 \pm 0.04) \times 10^{51} \mathrm{erg}$, respectively. Following Prentice et al. (2016), an amount of $0.055 \pm 0.006 M_{\odot}$ of ${ }^{56} \mathrm{Ni}$ is synthesised, corresponding to a peak luminosity of $(1.19 \pm 0.08) \times$ $10^{42} \mathrm{erg} \mathrm{s}^{-1}$.

\section{SPECTRAL STUDIES OF SN 2015AP AND SN 2016BAU}

In this section, we discuss spectral features of SN 2015ap and SN 2016bau and further compare their properties with other similar SNe. We modelled the spectra of these two SNe at different epochs using SYN++ (Branch et al. 2007; Thomas et al. 2011) and performed the spectral matching of the 12 , 13 , and $17 M_{\odot}$ spectral models given by Jerkstrand (2015) with the spectra of SN 2015ap and SN 2016bau at phases around $100 \mathrm{~d}$ past explosion. In this section, we also estimate the velocities of various lines present in the spectra, using their absorption troughs.

\subsection{Spectral properties of SN 2015ap}

The top and bottom panels of Figure 10 show the early phases $(-7 \mathrm{~d}$ to $+55 \mathrm{~d})$ and late phases $(+61 \mathrm{~d}$ to $+147 \mathrm{~d})$ spectral evolution, respectively. It is quite evident from the top panel of Figure 10 that initially SN 2015ap shows broadlined features (at $-7 \mathrm{~d}$ and $-2 \mathrm{~d}$ ), which later evolve to spec-

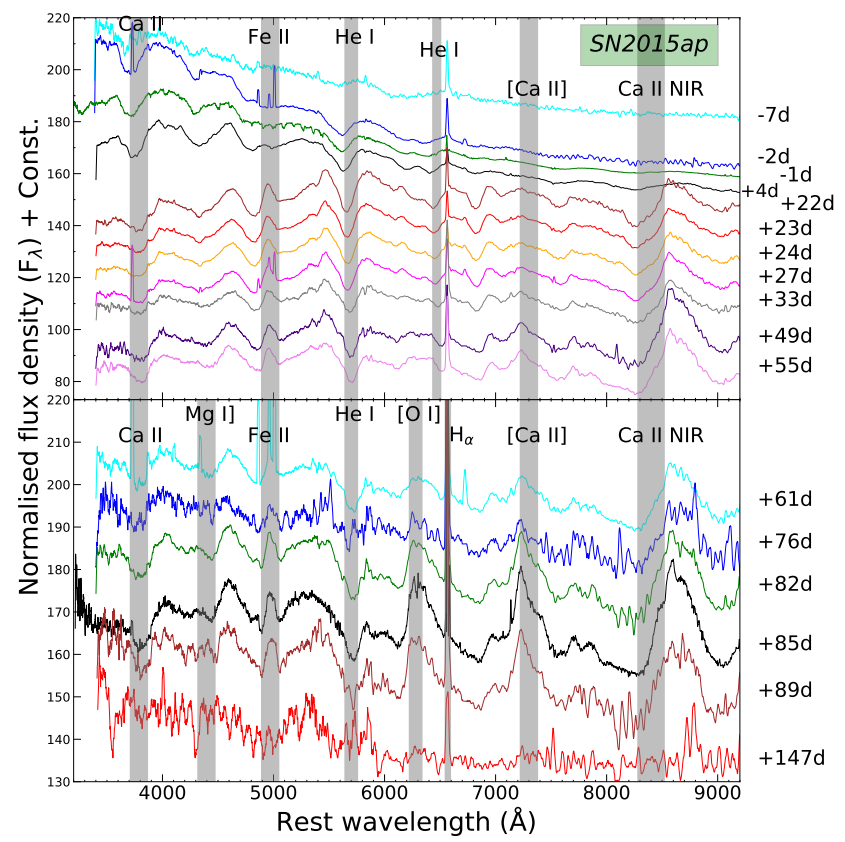

Figure 10. Top panel: the early-phase spectra up to $+55 \mathrm{~d}$. Bottom panel: the spectral evolution beyond $+55 \mathrm{~d}$ since $B_{\max }$ of SN 2015ap.

tra of a normal Type Ib SN. The characteristic He I line at $5876 \AA$ of a typical SN Ib is clearly seen in the spectra of SN 2015ap. We also see unambiguous He I features at $6678 \AA$ and $7065 \AA$, but these two lines are not as prominent as the one at $5876 \AA$. In the very early phases (up to $+4 \mathrm{~d}$ ), the He I line at $7065 \AA$ is hard to identify. We see that the He I absorption at $5876 \AA$ is stronger than any other absorption features, and it is present in every spectrum up to $+147 \mathrm{~d}$. The Fe II feature near $5169 \AA$ is very hard to be identified, and it seems to be highly blended with He I $5016 \AA$.

The spectral evolution also shows the Ca II nearinfrared (NIR) feature, which is almost absent in the very early phases $(-7 \mathrm{~d}$ to $+4 \mathrm{~d})$, but starts to develop very strongly from $+22 \mathrm{~d}$ onward. It is so strong that each spectrum on and after $+22 \mathrm{~d}$ shows it, even the lowest-SNR spectrum at $+147 \mathrm{~d}$. The forbidden [Ca II] feature near $7300 \AA$ is almost absent at very early phases $(-7 \mathrm{~d}$ to $+4 \mathrm{~d})$, then begins to develop very obviously in the spectra from $+22 \mathrm{~d}$, and is present up to $+89 \mathrm{~d}$. This [Ca II] feature can also get blended with [O II] emission at $7320 \AA$ and $7330 \AA$. It almost disappears in the spectrum at $+147 \mathrm{~d}$, though its absence may not be real owing to the very poor SNR of that spectrum. We also see the Ca II H\&K feature near $3934 \AA$, which is present in every spectrum. From the bottom panel of Figure 10, we see that as the spectra evolve, the absorption features of different lines tend to disappear and the emission features become more prominent. We see a very weak semiforbidden Mg I] line, at $4571 \AA$. However we can clearly see the forbidden emission lines of [O I] and [Ca II], which indicates the onset of the nebular phase. 


\subsubsection{Spectral comparison}

To investigate the spectroscopic behaviour of SN 2015ap, we compare its spectral features with those of other wellstudied SNe Ib such as SN 2004gq (Modjaz et al. 2014), SN 2008D (Modjaz et al. 2014), SN 2012au (Pandey et al. 2020), SN 2009jf (Sahu et al. 2011; Modjaz et al. 2014), iPTF13bvn (Srivastav et al. 2014a), SN 2007uy (Modjaz et al. 2014; Milisavljevic et al. 2010), SN 2007gr (Valenti et al. 2008; Modjaz et al. 2014), and SN 2005bf (Modjaz et al. 2014).

The top panel of Figure 11 shows the early phase $(-7 \mathrm{~d})$ spectral comparison of SN 2015ap with these well-studied SNe Ib. The spectral features of SN 2015ap look similar to those of SN 2008D and SN 2012au, compared to other $\mathrm{SNe} \mathrm{Ib}$. We can see that the Ca II H\&K feature, the Mg II feature, and the He I P Cygni profile match very well with those of SN 2008D, while in other SNe in the comparison sample, these features are much more developed. The blue end of the spectrum matches nicely with SN 2008D, while the redder part is featureless and much closer to SN 2012au and SN 2005bf. The He I $5876 \AA$ feature of SN 2015ap is completely different from that of SN 2007uy, but resembles that of SN 2008D and seems to be less evolved compared to that of SN 2004gq, SN 2012au, SN 2009jf, and iptf13bvn. The Fe II profile in SN 2015ap is hard to detect, which may be due to a very high initial optical opacity. We try to estimate the velocities using the various absorption features. As the spectrum at this epoch is continuum dominated, only a few absorption features were visible. The velocity estimated using He I absorption lines is $\sim 14,100 \mathrm{~km} \mathrm{~s}^{-1}$.

Further, we compare the +33 d spectrum of SN 2015ap with our comparison sample (middle panel of Figure 11). At this epoch, the spectrum of SN 2015ap shows various $\mathrm{P}$ Cygni profiles. We see that it almost exactly replicates the $+22 \mathrm{~d}$ spectrum of SN 2008D. The blended He I and Fe II profiles near $5016 \AA$ well match those of other $\mathrm{SNe} \mathrm{Ib}$, except for SN 2007uy, where the peak is almost absent, and for SN 2005bf, where it is double peaked. The He I feature at $5876 \AA$ matches well with other SNe Ib except for the cases of SN 2007uy and SN 2005bf, where the absorption features seem to be much broader. Here, once again, SN 2007uy seems to match much less and SN 2008D seems to best match the $+22 \mathrm{~d}$ spectrum of SN 2015ap. The velocities estimated using the Ca II NIR triplet and He I absorption features are $\sim 7800 \mathrm{~km} \mathrm{~s}^{-1}$ and $10000 \mathrm{~km} \mathrm{~s}^{-1}$, respectively.

For a much clear comparison, we compared the $+61 \mathrm{~d}$ spectrum of SN 2015ap (bottom panel of Fig. 11) with spectra of other well-studied $\mathrm{SNe} \mathrm{Ib}$. At this epoch also, the spectrum is still much closer to that of SN 2008D compared to other SNe Ib. The blended Fe II and He I feature near $5016 \AA$ shows an almost similar profile to that of SN 2008D. However, the $5876 \AA$ He I profile is narrower in SN 2015ap compared to SN 2008D. We can see the onset of the appearance of the [O I] line in SN 2015ap, which differs from SN 2008D. The He I profile is well matched in the cases of SN 1999dn and SN 2009jf, but the Ca II NIR triplet of these SNe differs from that of SN 2015ap. Here the velocities estimated using the Ca II NIR triplet and He I absorption features are $\sim 6900 \mathrm{~km} \mathrm{~s}^{-1}$ and $8100 \mathrm{~km} \mathrm{~s}^{-1}$, respectively.

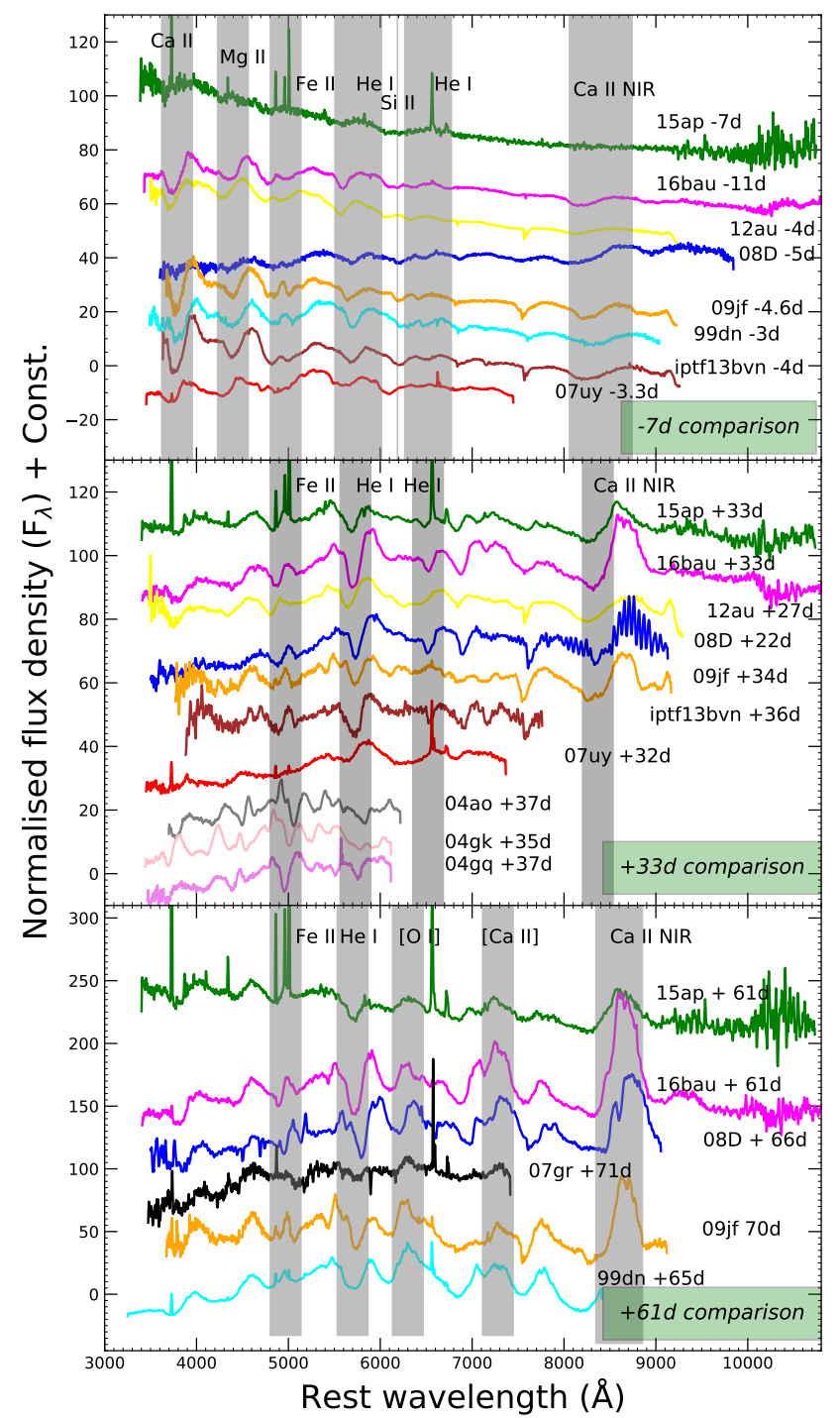

Figure 11. Spectral comparison of the spectra of SN 2015ap and SN 2016bau at epochs $-7 \mathrm{~d}$ (top panel), +33d (middle panel), and $+61 \mathrm{~d}$ (bottom panel). Various important SN Ib features have been compared with those of other similar-type SNe.

\subsubsection{Spectral modelling}

After confidently identifying the features present in the spectra of SN 2015ap and comparing them with those of other well-studied SNe, we tried to model a few spectra at various epochs using SYN++. The top panel in Figure 12 shows the early-phase $(-7 \mathrm{~d})$ spectrum of SN 2015ap. This spectrum displays weak and broad P Cygni profiles of He I, Ca II H\&K, and the Ca II NIR triplet, and also some blended features of Fe II. We also show the best-matching synthetic spectrum, generated by SYN++. The absorption features due to Ca II H\&K, Ca II NIR triplet, He I, and Fe II multiplets are easily reproduced. The photospheric velocity and blackbody temperature associated with the best-fit spectrum are $12,000 \mathrm{~km} \mathrm{~s}^{-1}$ and $12,000 \mathrm{~K}$, respectively. We perform SYN++ matching to four additional spectra from epochs $-2 \mathrm{~d}$ to +33 d (Fig. 12). With the passage of time, the SN expands, 


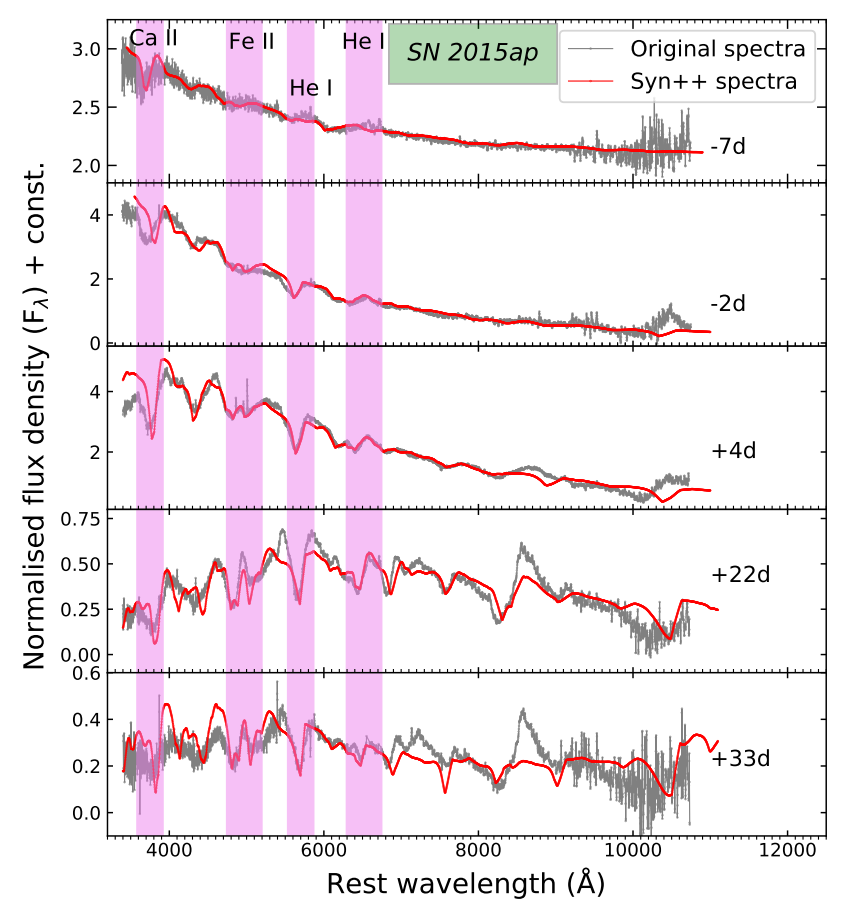

Figure 12. SYN++ modelling of the spectra of SN 2015ap at epochs $-7 \mathrm{~d},-2 \mathrm{~d},+4 \mathrm{~d},+22 \mathrm{~d}$, and $+33 \mathrm{~d}$. Prominent He I features could be produced nicely.

cools gradually, and its expansion velocity decreases slowly, so we see a gradual decrease in the values of these fitted parameters. The photospheric velocities during the phase of $-7 \mathrm{~d}$ to $+33 \mathrm{~d}$ vary from $13,000 \mathrm{~km} \mathrm{~s}^{-1}$ to $6800 \mathrm{~km} \mathrm{~s}^{-1}$, and the blackbody temperature varies from $12,000 \mathrm{~K}$ to $4500 \mathrm{~K}$, which are in good agreement with those obtained photometrically from blackbody fits. Owing to the local thermodynamic equilibrium (LTE) approximation, SYN++ does not work well for the later epochs and cannot be used to fit the spectra.

To get an estimate of the progenitor mass, we used the +98.75 d post-explosion spectrum of SN 2015ap and plotted it along with the 12,13 , and $17 M_{\odot}$ model spectra from Jerkstrand (2015) at $100 \mathrm{~d}$, after scaling by a factor of $\exp (-2 \times \Delta t / 111.4)$ (Jerkstrand 2015), where $\Delta t=1.25$, is the time difference between the epoch of the model spectrum and the epoch of the observed spectrum. We can see that the $12 M_{\odot}$ and $17 M_{\odot}$ model spectra seem to reproduce the observed spectrum of SN 2015ap. The $12 M_{\odot}$ spectrum very nicely matches the observed spectrum throughout the entire wavelength range, while the $17 M_{\odot}$ spectrum slightly overproduces the flux near the Ca II NIR triplet close to $8500 \AA$ A. However, the $13 M_{\odot}$ model spectrum fails to explain the observed fluxes throughout the entire wavelength range. Thus, based on this analysis, a range of $12-17 M_{\odot}$ is expected for the possible progenitor mass of SN 2015ap, in agreement with that described by Gangopadhyay et al. (2020).

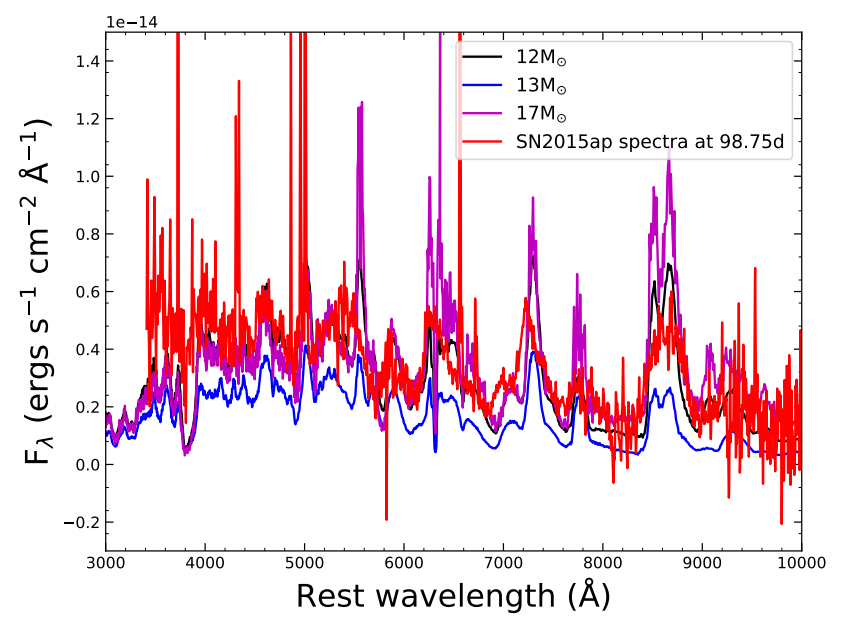

Figure 13. The $t=+98.75 \mathrm{~d}$ spectrum of SN 2015ap plotted along with the 12,13 , and $17 M_{\odot}$ models from Jerkstrand (2015) at $100 \mathrm{~d}$ scaled with an exponential factor $\exp (-2 \times 1.25 / 111.4)$. The $12 M_{\odot}$ and $17 M_{\odot}$ models seem to best match the observed spectrum of SN 2015ap.

\subsubsection{Velocity evolution of various lines of SN 2015 ap}

We used the blue-shifted absorption minima of P Cygni profiles and the special-relativistic Doppler formula (Sher 1968) to obtain the velocities of several lines. Figure $14 a$ shows the He I and the Ca II NIR triplet velocity evolution of SN 2015ap. In the initial few days, the line velocities tend to decrease rapidly. At an epoch of $+24 \mathrm{~d}$, the velocities estimated using He I $5876 \AA$ and the Ca II NIR triplet are $\sim 10,600 \mathrm{~km} \mathrm{~s}^{-1}$ and $8200 \mathrm{~km} \mathrm{~s}^{-1}$, respectively. The velocities estimated using these two lines drop to $\sim 9100 \mathrm{~km} \mathrm{~s}^{-1}$ and $7100 \mathrm{~km} \mathrm{~s}^{-1}$ (respectively) at an epoch of $+49 \mathrm{~d}$. In the late phases, the velocities decline gradually.

Figure $14 c, d$, show comparisons of velocities obtained using the Ca II NIR triplet and He I $5876 \AA$ features with other well-studied SNe. Initially the Ca II NIR line velocity of SN 2015ap evolves in a manner similar to iptf13bvn, thereafter it decays slowly, attaining a velocity of $\sim 7600 \mathrm{~km} \mathrm{~s}^{-1}$ at +89 d. We see that the ejecta velocity of SN 2015ap obtained using He I $5876 \AA$ is higher than that of other SNe Ib but closer to SN 2008D.

\subsection{Spectral properties of SN 2016bau}

Figure 15 shows the spectral evolution of SN 2016bau for a period of $-14 \mathrm{~d}$ to $+107 \mathrm{~d}$. In the first spectrum $(-14 \mathrm{~d})$, we see well-developed He I features (5876, 6678, and $7065 \AA$ ). Also, the spectrum on this particular epoch displays a weak and broad Ca II NIR feature, but with the passage of time (beyond $\sim+33 \mathrm{~d}$ ), prominent Ca II NIR features start to appear. We also see that in the initial phases there is a strong Ca II H \& K feature, but it becomes progressively weaker at later phases. As the phase approaches the date of $B$-band maximum brightness, the He I features start to appear much more strongly, as seen in the spectra at $-11 \mathrm{~d},+4 \mathrm{~d}$, and $+18 \mathrm{~d}$. Beyond $-11 \mathrm{~d}$, the longer wavelengths exhibit strong Ca II NIR features. The spectra also display very strong P Cygni profiles of He I at $5016 \AA$ after $t=-11 \mathrm{~d}$. The 


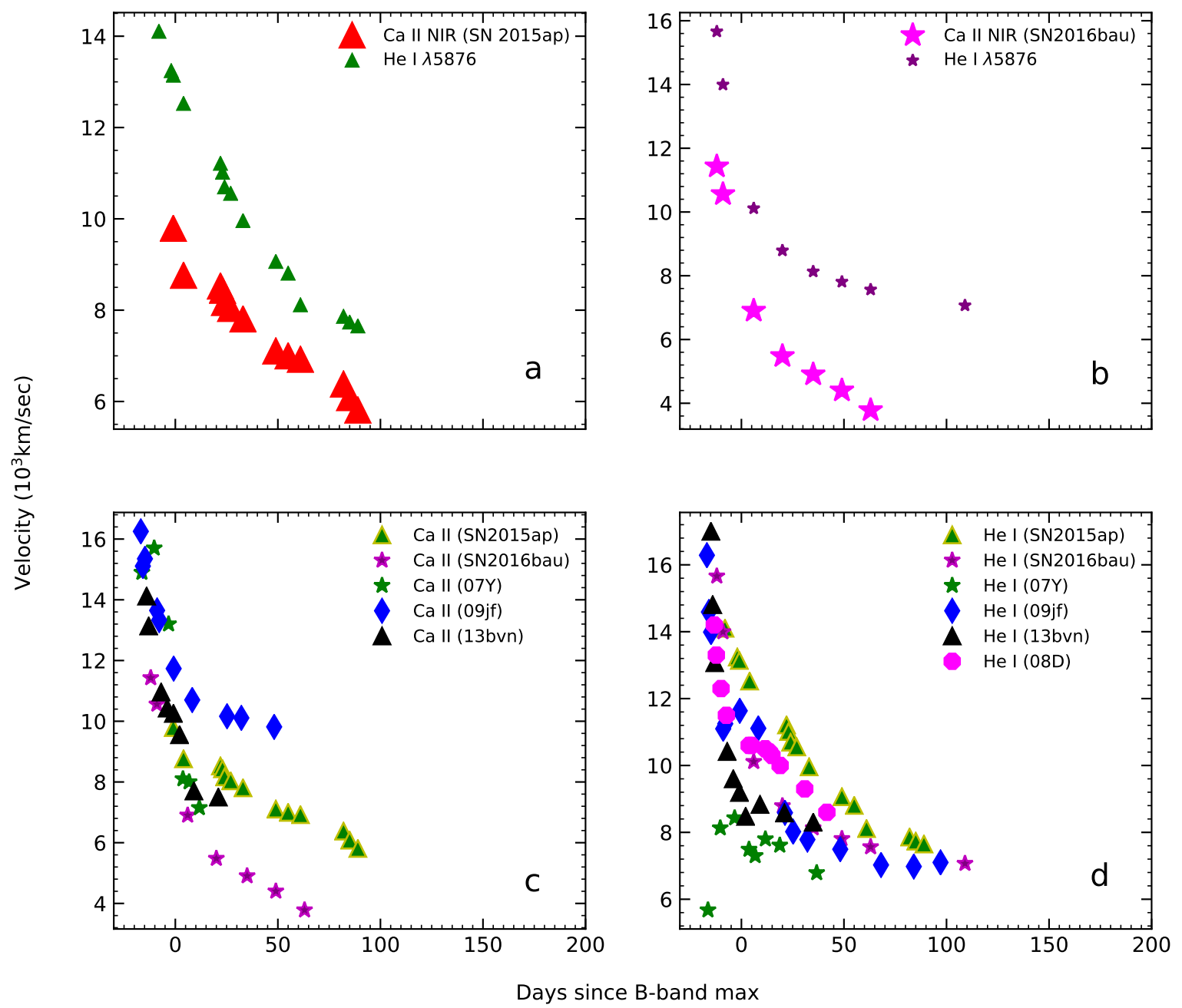

Figure 14. (a) The temporal evolution of velocities of He I $5876 \AA$ and the Ca II NIR triplet for SN 2015ap. (b) Same as (a) but for SN 2016bau. The bottom two panels show the comparison of these line velocities of SN 2015ap and SN 2016bau with SNe 2007Y (Stritzinger et al. 2009), 2009jf (Sahu et al. 2011), iPTF13bvn (Srivastav et al. 2014a), and 2008D (Modjaz et al. 2014).

emergence of very strong features of He I in the early-time spectra confirms this SN to be of Type Ib.

\subsubsection{Spectral comparison}

To investigate the spectroscopic behaviour of SN 2016bau, we have compared its spectral features with those of other well-studied SNe Ib. We used a sample similar to that for SN 2015ap. The top panel of Figure 11 shows the earlyphase (-11d) spectral comparison of SN 2016bau with other well-studied H-stripped CCSNe. The spectral features of SN 2016bau look much more similar to those of SN 2012au, SN 2009jf, and SN 1999dn compared to other SNe. we can see that the Ca II H\&K feature, the Mg II feature, and the He I P Cygni profile of SN 2016bau match very well those of SN 2012au, SN 2009jf, and SN 1999dn. The He I $5876 \AA$ feature of SN $2016 \mathrm{bau}$ is completely different from that of SN 2007uy, SN 2015ap, and SN2008D. As with SN 2015ap, the blended Fe II profile in SN 2016bau is hard to detect, which may be due to a very high initial optical opacity. The spectrum at this epoch has nicely developed He I features. The velocity estimated using the He I $5876 \AA$ absorption line is $\sim 15,600 \mathrm{~km} \mathrm{~s}^{-1}$.

The middle panel of Figure 11 shows the $+33 \mathrm{~d}$ spectral comparison of SN 2016bau with other well-studied SNe Ib. The spectrum at this epoch contains many $\mathrm{P}$ Cygni profiles of various lines. These spectral features look most similar to those of SN 2008D and SN 2015ap, compared to other $\mathrm{SNe} \mathrm{Ib}$. We see that the Ca II NIR feature, the Fe II feature, and the He I P Cygni profile match very 


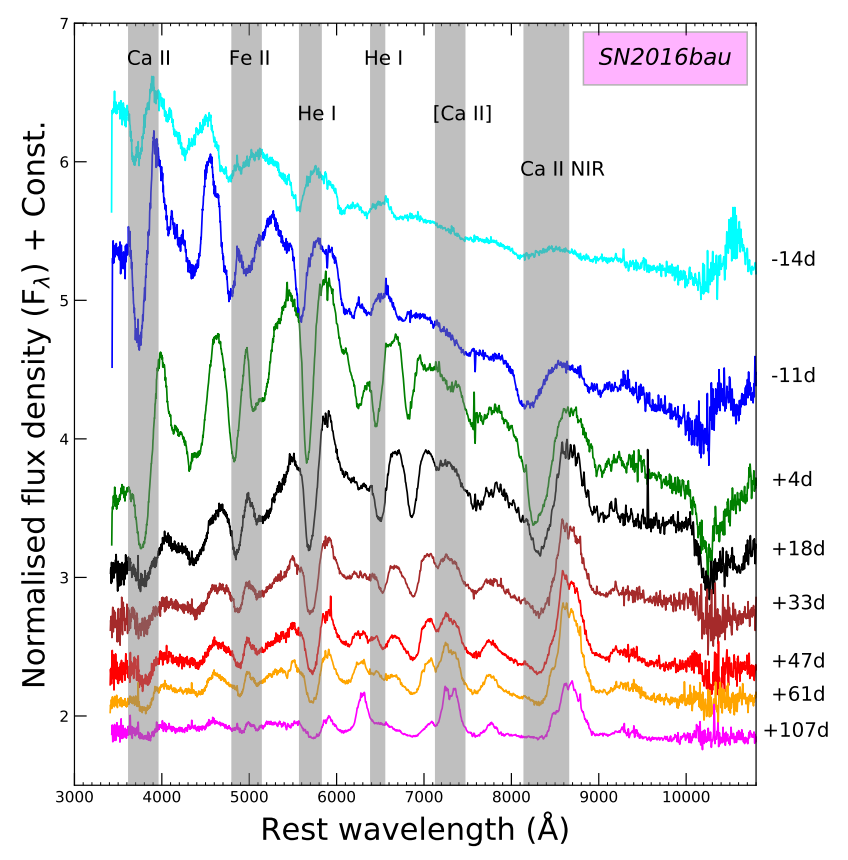

Figure 15. Various line identifications in the overall spectral evolution of SN 2016bau. Strong He I features along with other important lines are indicated.

well those of SN 2008D and SN 2015ap, compared to other SNe. SN 2009jf also seems to match nicely in the redder part of the spectrum. The He I $5876 \AA$ feature of SN 2016bau is completely different from those of SN 2007uy, SN 2005bf, SN 2004gq, and SN 2007gr, but resembles those of SN 2008D, SN 2015ap, and SN2009jf. The He I $5876 \AA$ profile of SN 2016bau seems to be more asymmetric and narrower than that of iptf13bvn. At this epoch, the velocities estimated using the absorption features of $\mathrm{He} \mathrm{I}$ and the Ca II NIR triplet are $\sim 8100 \mathrm{~km} \mathrm{~s}^{-1}$ and $4900 \mathrm{~km} \mathrm{~s}^{-1}$, respectively.

For much clearer comparisons, we also compared the +61 d spectrum of SN 2016bau (bottom panel of Figure. 11) with other well-studied H-stripped CCSNe spectra. At this epoch, the bluer part of the spectrum is much closer to SN 2008D compared to other SNe Ib. Here the blended Fe II and He I features near $5016 \AA$ show profiles nearly similar to those of SN 2008D and SN 2009jf. However, the $5876 \AA$ Ae I profile is broader in SN 2016bau compared with SN 2008D. We can see that the onset of the appearance of $[\mathrm{O} \mathrm{I}]$ in SN 2016bau is slightly different from that of SN 2008D, while it matches nicely that of SN 2009jf. The He I profile is well matched in the case of SN 2008D. Here the velocities estimated using the Ca II NIR and He I absorption features are $\sim 3700 \mathrm{~km} \mathrm{~s}^{-1}$ and $7600 \mathrm{~km} \mathrm{~s}^{-1}$, respectively.

\subsubsection{Spectral modelling}

After confidently identifying various spectral features, we tried to model the spectra of SN 2016bau at different epochs using SYN++. The top panel of Figure 16 shows the earlyphase $(-14 \mathrm{~d})$ spectrum of SN 2016bau. It contains many broad P Cygni profiles of He I, Ca II H\&K, the Ca II NIR

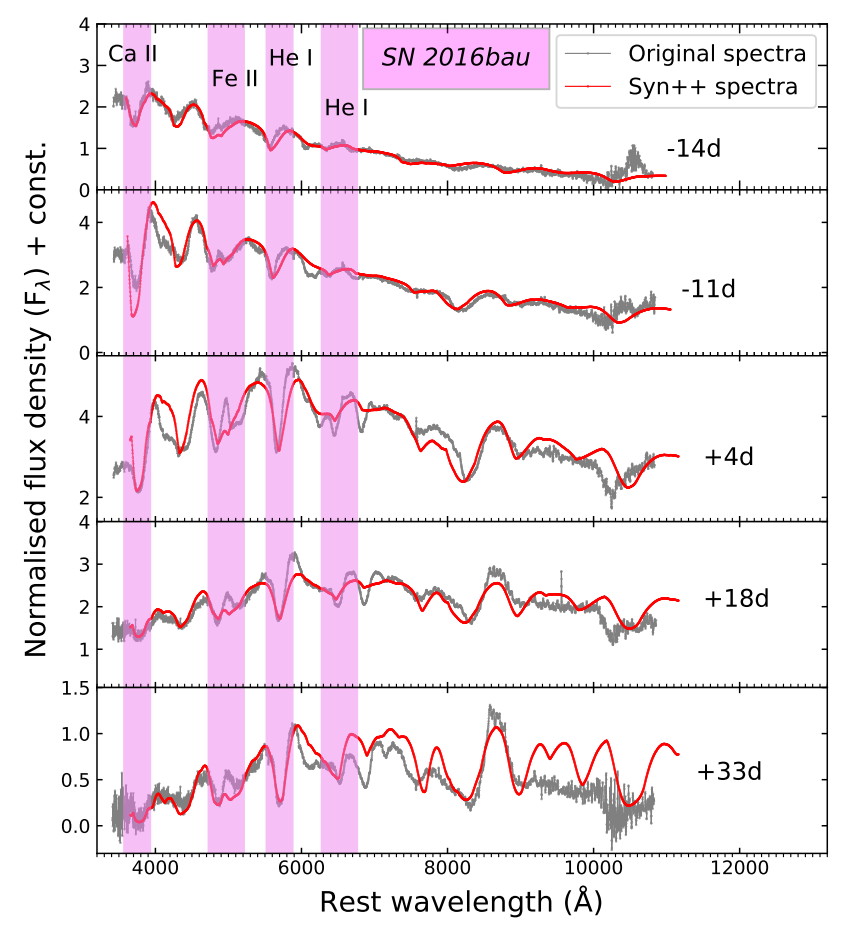

Figure 16. SYN++ modelling of the spectra of SN 2016bau at epochs $-14 \mathrm{~d},-11 \mathrm{~d},+4 \mathrm{~d},+18 \mathrm{~d}$, and $+33 \mathrm{~d}$.

triplet, and also some blended features of Fe II. In this figure we have also presented the best-matching synthetic spectrum generated by SYN++. The modelled spectrum easily reproduces the absorption features of Ca II H\&K, Ca II NIR, He I, and the Fe II multiplet. The photospheric velocity and blackbody temperature associated with the best-fit spectrum are $16,000 \mathrm{~km} \mathrm{~s}^{-1}$ and $9000 \mathrm{~K}$ respectively. We performed SYN++ matching for four additional spectra which covers a period of $-11 \mathrm{~d}$ to $+33 \mathrm{~d}$ (subsequent panels of Fig. 16). With the passage of time, the SN expands and cools gradually, and its expansion velocity decreases slowly, so we see a gradual decrease in the fit parameters such as velocity and temperature in the later phases. The photospheric velocity during the phase of $-14 \mathrm{~d}$ to $+33 \mathrm{~d}$ varies from $16,000 \mathrm{~km} \mathrm{~s}^{-1}$ to $8000 \mathrm{~km} \mathrm{~s}^{-1}$ and the blackbody temperature ranges from $9000 \mathrm{~K}$ to $4000 \mathrm{~K}$, in good agreement with values obtained photometrically from blackbody fits.

We also try to match the $+121 \mathrm{~d}$ spectrum of SN 2016bau with the $12 M_{\odot}, 13 M_{\odot}$, and $17 M_{\odot}$ model spectra at $+100 \mathrm{~d}$ from Jerkstrand (2015), scaled with a factor of $\exp (-2 \times \Delta t / 111.4)$ (Jerkstrand 2015), where $\Delta t=$ 21 , is the time difference of the epoch of model spectrum and the epoch of observed spectrum. We can see that all three models over-predict the observed fluxes from $3000 \AA$ to around $6000 \AA$, beyond which the $12 M_{\odot}$ model spectrum seems to best describe the observed spectrum. It could nicely explain the [Ca II] emission near $7300 \AA$ and the Ca II NIR feature near $8500 \AA$. The $13 M_{\odot}$ model spectrum also produces the $[\mathrm{Ca} \mathrm{II}]$ emission but fails to explain the observed fluxes near the Ca II-NIR triplet. The $17 M_{\odot}$ model spectrum overpredicts the flux throughout the entire wavelength range and thus fails to explain the spectrum of SN 2016bau. 


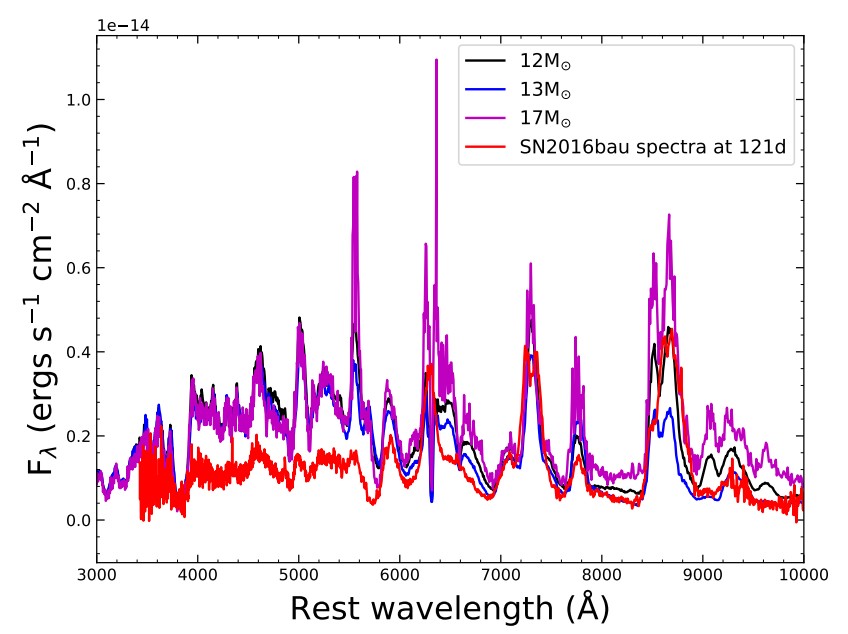

Figure 17. The $t=+121 \mathrm{~d}$ spectrum of SN 2016bau plotted along with the 12,13 , and $17 M_{\odot}$ models from Jerkstrand (2015) at $100 \mathrm{~d}$ scaled with an exponential factor $\exp (-2 \times 21 / 111.4)$. The $12 M_{\odot}$ model seems to best match the spectrum of SN $2016 \mathrm{bau}$.

Hence, based on our analysis, a slightly low-mass progenitor $\left(\leq 12 M_{\odot}\right)$ is expected.

\subsubsection{Velocity evolution of Various lines of SN 2016bau}

We used the blue-shifted absorption minima of P Cygni profiles to obtain the velocities of He I and Ca II NIR lines. Figure $14 b$ shows the He I and Ca II NIR velocity evolution of SN 2016bau. In the initial few days, the line velocities tend to decrease rapidly, but in later phases, the velocities decline gradually. At an early epoch of $-14 \mathrm{~d}$, the velocities estimated using He I $5876 \AA$ and the Ca II NIR triplet are $\sim 15,600 \mathrm{~km} \mathrm{~s}^{-1}$ and $\sim 11,400 \mathrm{~km} \mathrm{~s}^{-1}$, respectively. The velocities estimated using these two lines drop to $\sim 7800 \mathrm{~km} \mathrm{~s}^{-1}$ and $\sim 4400 \mathrm{~km} \mathrm{~s}^{-1}$ (respectively) at an epoch of $+47 \mathrm{~d}$. Beyond $+47 \mathrm{~d}$, the velocities continue to decline with a slower rate as compared to initial decline rate. Figure $14 c, d$ show the comparison of these two line velocities with other well-studied SNe. Initially, the Ca II NIR velocity declines very fast but in the later epochs, only gradual decline is seen. It evolves in a manner very similar to iPTF13bvn, but in the late phases, the velocities are slower than other SNe. The velocity obtained using He I $5876 \AA$ of SN 2016bau evolves in a manner similar to that of other SNe Ib, but much closer to SN 2009jf and iPTF13bvn. The He I line velocity of SN 2016bau at $+33 \mathrm{~d}$ reaches $\sim 8200 \mathrm{~km} \mathrm{~s}^{-1}$, nearly equal to those of SN 2009jf and iPTF13bvn.

\section{MINIM MODELLING OF THE QUASI-BOLOMETRIC LIGHT CURVE}

In this section, we fit the radioactive decay (RD) and the magnetar (MAG) powering mechanisms, as discussed by Chatzopoulous et al. 2013 (see also Wheeler et al. 2017; Kumar et al. 2020, 2021), by employing the MINIM (Chatzopoulous et al. 2013) code. MINIM is a $\chi^{2}$-minimisation fit-

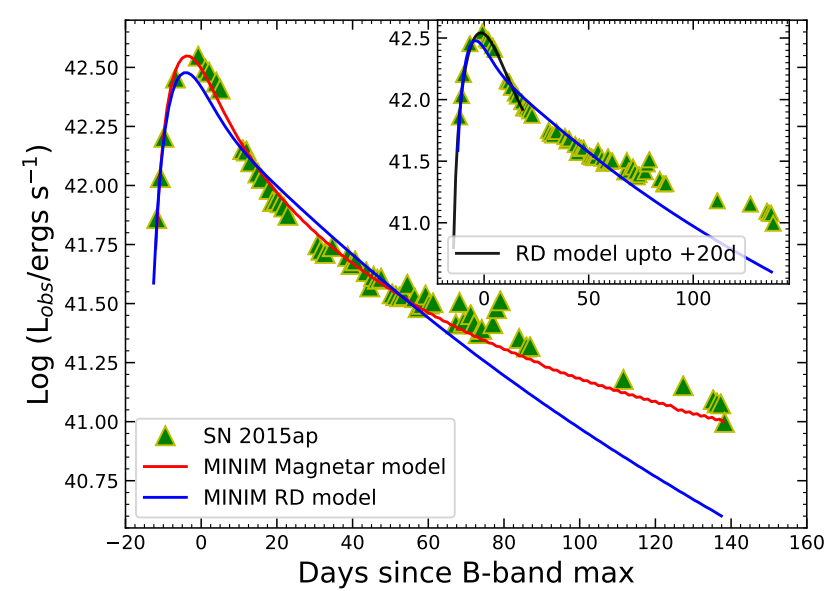

Figure 18. MINIM modelling of the quasi-bolometric light curve of SN 2015ap. The inset shows the RD model fitting only for the early phase (up to $+20 \mathrm{~d}$ ).

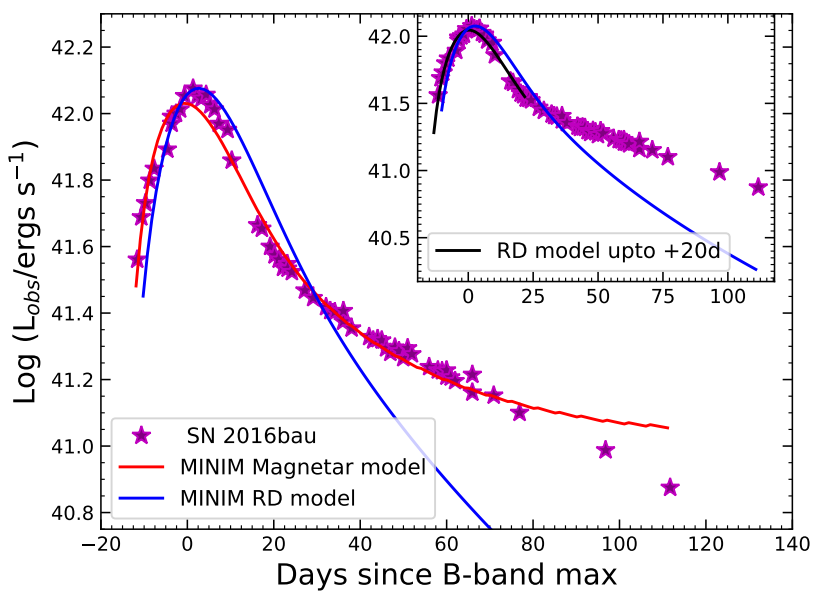

Figure 19. MINIM modelling of the quasi-bolometric light curve of SN 2016bau. The figure in the inset shows the RD model fitting only for the early phase (up to $+20 \mathrm{~d}$ ).

ting code that utilises the Price algorithm (Brachetti et al. 1997). In the RD model, the radioactive decay of ${ }^{56} \mathrm{Ni}$ and ${ }^{56} \mathrm{Co}$ leads to the deposition of energetic gamma-rays that are assumed to thermalise in the homologously expanding SN ejecta and thus powering the light curve. In the MAG model, the light curves are powered by the energy released by the spin-down of a young magnetar, located in the centre of the SN ejecta. Following Prentice et al. (2019), we have adopted a constant opacity, $\kappa=0.07 \mathrm{~cm}^{2} \mathrm{~g}^{-1}$, for both SN 2015ap and SN 2016bau.

\subsection{SN 2015ap}

Figure 18 shows the results of RD and MAG model fittings to the quasi-bolometric light curve of SN 2015ap. All of the fitted and calculated parameters are listed in Tables 1 and 2. The ejecta mass $\left(M_{\mathrm{ej}}\right)$ in the RD and MAG models was calculated using Equation 1 from Wheeler et al. (2015). Al- 
Table 1. Minimum $\chi^{2} /$ dof parameters for SN 2015ap and SN 2016bau for the RD model.

\begin{tabular}{cccccc}
\hline \hline & $\begin{array}{c}M_{\mathrm{Ni}}{ }^{a} \\
\left(M_{\odot}\right)\end{array}$ & $\begin{array}{c}t_{\mathrm{d}}{ }^{b} \\
\text { (days) }\end{array}$ & $A_{\gamma^{c}}$ & $\begin{array}{c}M_{\mathrm{ej}}{ }^{b} \\
\left(M_{\odot}\right)\end{array}$ & $\chi^{2 / \mathrm{dof}}$ \\
\hline \hline SN 2015ap & & & & & \\
\hline whole light curve & $0.094 \pm 0.004$ & $8.0 \pm 2.0$ & $30.05 \pm 1.05$ & $0.64 \pm 0.3$ & 6.2 \\
+20 d data & $0.181 \pm 0.006$ & $14.5 \pm 0.3$ & $5.3 \pm 0.3$ & $2.1 \pm 0.09$ & 1.2 \\
\hline SN 2016bau & & & & & \\
\hline whole light curve & $0.08 \pm 0.01$ & $20.5 \pm 0.7$ & $7.8 \pm 0.5$ & $2.8 \pm 0.2$ & 22.7 \\
+20 d data & $0.065 \pm 0.001$ & $18.09 \pm 0.3$ & $9.95 \pm 0.4$ & $1.81 \pm 0.07$ & 1.2 \\
\hline \hline
\end{tabular}

$a$, mass of ${ }^{56} \mathrm{Ni}$ synthesised; $b$, effective diffusion timescale,

$c$, optical depth for the $\gamma$-rays measured $10 \mathrm{~d}$ after the explosion; $d$, ejecta mass, with $\kappa=0.07 \mathrm{~cm}^{2} \mathrm{~g}^{-1}$.

Table 2. Minimum $\chi^{2}$ /dof parameters for SN 2015ap and SN 2016bau for the MAG model.

\begin{tabular}{|c|c|c|c|c|c|c|c|c|c|}
\hline & $\begin{array}{c}R_{0}{ }^{a} \\
\left(10^{13} \mathrm{~cm}\right)\end{array}$ & $\begin{array}{c}E_{p}^{b} \\
\left(10^{51} \mathrm{erg}\right)\end{array}$ & $\begin{array}{c}t_{d}^{c} \\
\text { (days) }\end{array}$ & $\begin{array}{c}t_{p}{ }^{d} \\
\text { (days) }\end{array}$ & $\begin{array}{c}v_{\exp }{ }^{e} \\
\left(10^{3} \mathrm{~km} \mathrm{~s}^{-1}\right)\end{array}$ & $\begin{array}{l}M_{\mathrm{ej}}{ }^{f} \\
\left(M_{\odot}\right)\end{array}$ & $\begin{array}{l}P_{i}^{g} \\
(\mathrm{~ms})\end{array}$ & $\begin{array}{c}B^{h} \\
\left(10^{14} \mathrm{G}\right)\end{array}$ & $\overline{\chi^{2} / \text { dof }}$ \\
\hline \multicolumn{10}{|l|}{ SN 2015ap } \\
\hline & $0.594 \pm 0.003$ & $0.01210 \pm 0.00007$ & $8.7 \pm 0.1$ & $12.06 \pm 0.08$ & $6.03 \pm 0.02$ & $0.75 \pm 0.02$ & $40.6 \pm 0.1$ & $25.5 \pm 0.2$ & 1.54 \\
\hline \multicolumn{10}{|l|}{ SN 2016bau } \\
\hline & $7.9 \pm 0.7$ & $0.00403 \pm 0.00007$ & $8.5 \pm 0.1$ & $15.1 \pm 0.1$ & $6.2 \pm 0.5$ & $1.26 \pm 0.02$ & $70.4 \pm 0.6$ & $52.6 \pm 0.8$ & 1.4 \\
\hline
\end{tabular}

$a$, progenitor radius; $b$, magnetar rotational energy; $c$, effective diffusion timescale (in days); $d$, magnetar spin-down timescale; $e$, SN expansion velocity; $f, \kappa=0.07 \mathrm{~cm}^{2} \mathrm{~g}^{-1}$ is used; $g$, initial period of the magnetar; $h$, magnetic field of the magnetar.

though the RD model seems to reasonably fit the observed quasi-bolometric light curve, it seems unable to reproduce not only the observed peak luminosity, but also the light curve at late phases, after $+50 \mathrm{~d}$. The nickel mass and ejecta mass obtained from these models are somewhat smaller than the ones inferred directly from the observed rise time and peak luminosity in Sec.3.1.3. As the RD model is the prominent powering mechanism for normal $\mathrm{SNe} \mathrm{Ib}$, we tried to fit the RD model only up to a relatively early phase $(\sim+20 \mathrm{~d})$. We see in the inset of Figure 18 that the early phase is nicely fitted in this case. A nickel mass $\left(M_{\mathrm{Ni}}\right)$ of $0.181 \pm 0.006 M_{\odot}$ and an ejecta mass $\left(M_{\mathrm{ej}}\right)$ of $2.1 \pm 0.09 M_{\odot}$ obtained through this fitting are close to the observed values listed in Sec.3.1.3. The fitted and calculated values from the photospheric phase are collected in Table 1.

The MAG model fits the whole observed light curve, both around peak brightness as well as during the late phase, better than the RD model. However, the fitted parameters seem to be unphysical, particularly the very slow initial rotation $\left(P_{i} \approx 40 \mathrm{~ms}\right)$ and the very low initial rotational energy $\left(E_{p} \approx 10^{49} \mathrm{erg}\right.$ ). This is not surprising given that the magnetar model contains two timescales, one for the rising part and another for the declining part of the light curve (Chatzopoulous et al. 2013). Thus, the possibility of SN 2015ap powered by spin-down of a magnetar is less likely.

\subsection{SN 2016bau}

Figure 19 shows the results of the RD and MAG model fittings to the quasi-bolometric light curve of SN 2016bau. The fitted and calculated parameters are listed in Tables 1 and 2. The RD model can fit the observed peak luminosity, but huge deviations from the observed light curve are seen in the later phases. The model also fails to match the observed stretch factor of the light curve. Like in the case of SN 2015ap, we also tried to fit only the early part, before $+20 \mathrm{~d}$ post-peak (see the inset in Fig. 19). In this case, the fitted parameters, such as the nickel mass of $0.065 \pm$
$0.002 M_{\odot}$ and the ejecta mass of $1.81 \pm 0.07 M_{\odot}$ (see Table 1), are very close to the observed values in Section 3.2.3. Similar to SN 2015ap, the slow rotation $\left(P_{i} \approx 70 \mathrm{~ms}\right)$, low magnetar rotational energy $\left(E_{p} \approx 10^{48} \mathrm{erg}\right)$, and very high progenitor radius $\left(\sim 1200 R_{\odot}\right)$ make the MAG model physically unrealistic for SN 2016bau, despite the better fit to the whole light curve in Figure 19.

\subsection{Summary of MINIM modelling}

The failure of the semi-analytical RD models to simultaneously fit the early and late parts of the light curves of SN 2015ap and SN 2016bau highlights the issues related to the validity of such kinds of models in the case of strippedenvelope SNe. The difficulty in explaining the late-phase decline rate with the assumed diffusion model have already been explored by Wheeler et al. (2015).

Figures 18 and 19 reveal another issue: the model light curve is steeper after $+30-40 \mathrm{~d}$ than the observed one. This indicates that the early- and late-phase data cannot be fitted simultaneously with the same model parameters. Even though the nickel mass of the second model fits (insets in Figs. 18 and 19) the observed peak luminosity, the decline rate, which is related to the ejecta mass (see Eq. 1 of Wheeler et al. (2015)) is clearly too fast, suggesting an underestimated $M_{\mathrm{ej}}$.

This issue is very probably related to the assumption of the constant ejecta density profile (and also the constant opacity). The less steep late part of the light curve needs more ejecta mass to trap the heating gamma-rays originating from the Ni and Co decay. The early part, however, suggests ejecta that dilute much faster than what can fit the late part. Within the context of the constant-density model, this dichotomy means that fitting only the early part results in a lower ejecta mass compared to fitting only the late part (Fig.20).

Since this issue cannot be solved self-consistently with the constant-density model, and it can plague the mass es- 


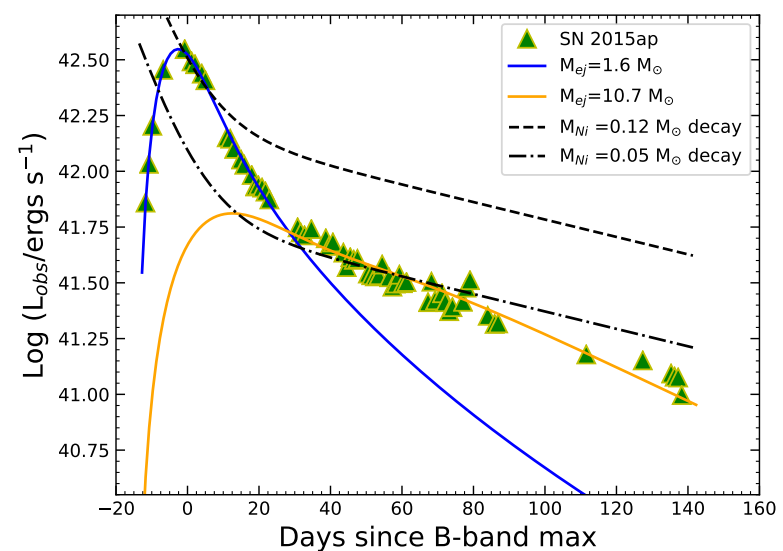

Figure 20. Detailed MINIM modelling to the quasi-bolometric light curve of SN 2015ap. All of the models with different $M_{\mathrm{ej}}$ and $M_{\mathrm{Ni}}$ are shown together. The models with $M_{\mathrm{ej}}=1.6 M_{\odot}$ and $10.7 M_{\odot}$ show the RD model fittings considering only the early- and latephase data, respectively. The other two models show the effect of the amount of nickel present.

timates that consider only the early or late part of the light curve, we investigate it further in the following sections by applying more realistic models for the progenitors and the SN light curves.

\section{MESA MODELLING OF A $12 M_{\odot}$ ZAMS PROGENITOR STAR}

Adoption of a $12 M_{\odot}$ ZAMS progenitor star for SN 2015ap is primarily based on the results of comparison of the three models from Jerkstrand (2015) and also in the literature; in addition, it supports the observed amount of ejecta mass for SN 2015ap. The ejecta mass for SN 2016bau was calculated to be $\sim 1.6 M_{\odot}$. On account of such an ejecta mass and the result of Jerkstrand (2015) spectral model matching, we also choose a $12 M_{\odot}$ ZAMS star as a possible progenitor for SN 2016bau.

We first evolve the $12 M_{\odot}$ ZAMS star until the onset of core-collapse, using the one-dimensional stellar evolution code MESA, version 11701(Paxton et al. 2011, 2013, $2015,2018)$. We do not consider rotation and assume an initial metallicity of $Z=0.02$. Convection is modelled using the mixing theory of Henyey et al. (1965), adopting the Ledoux criterion. We set the mixing-length parameters to $\alpha=3.0$ in the region where the mass fraction of hydrogen is greater than 0.5 , and set it to 1.5 in the other regions. Semi-convection is modelled following Langer et al. (1985) with an efficiency parameter of $\alpha_{\mathrm{sc}}=0.01$. For the thermohaline mixing, we follow Kippenhahn et al. (1980), and set the efficiency parameter as $\alpha_{\text {th }}=2.0$. We model the convective overshooting with the diffusive approach of Herwig (2000), with $f=0.01$ and $f_{0}=0.004$ for all the convective core and shells. We use the "Dutch" scheme for the stellar wind, with a scaling factor of 1.0. The "Dutch" wind scheme in MESA combines results from several papers. Specifically, when $T_{\text {eff }}>10^{4} \mathrm{~K}$ and the surface mass fraction of hydro- gen is greater than 0.4, the results of Vink et al. (2001) are used, and when $T_{\text {eff }}>10^{4} \mathrm{~K}$ and the surface mass fraction of hydrogen is less than 0.4, the results of Nugis \& Lamers (2000) are used. In the case when $T_{\text {eff }}<10^{4} \mathrm{~K}$, the de Jager et al. (1988) wind scheme is used.

$\mathrm{SNe} \mathrm{Ib}$ have been considered to originate from massive stars which lose almost all of their hydrogen envelope, most probably due to binary interaction (e.g., Yoon et al. 2010; Dessart et al. 2012; Eldridge \& Maund 2016; Ouchi \& Maeda 2017). Here, in order to produce such a stripped model, we artificially strip the hydrogen envelope, mimicking the binary interaction. Specifically, after evolving the model until the exhaustion of helium, we impose an artificial mass-loss rate of $\dot{M} \gtrsim 10^{-4} M_{\odot} \mathrm{yr}^{-1}$ until the total hydrogen mass of the star goes down to $0.01 M_{\odot}$. After the hydrogen mass reaches the specified limit, we switch off the artificial mass loss and evolve the model until the onset of core-collapse. At the time of core-collapse, our model has a total mass of $3.42 M_{\odot}$.

\section{EXPLOSIONS OF MODELLED PROGENITORS USING SNEC AND STELLA}

In this section we briefly discuss the assumptions and setups to produce artificial explosions using SNEC (Morozova et al. 2015) and STELLA (Blinnikov et al. 1998, 2000; Branch et al. 2006) for SN 2015ap and SN 2016bau.

\subsection{SN 2015ap}

Using the progenitor model on the verge of core-collapse obtained through MESA, we then carried out the radiation hydrodynamical simulations. For this purpose, we use the publicly available codes SNEC and STELLA.

SNEC is a one-dimensional Lagrangian hydrodynamic code, which also solves radiation energy transport with the flux-limited diffusion approximation. The code generates the bolometric light curve and the photospheric velocity evolution of the SN, along with other observed parameters. The setup for the calculation using SNEC closely follows Ouchi \& Maeda (2019). Here, we briefly summarise the important parameters and modifications made to Ouchi \& Maeda (2019). First, we excise the innermost $1.4 M_{\odot}$ before the explosion, assuming that it collapses to form a neutron star. The number of cells is set to be 70. Although this number is relatively small, we have confirmed that the light curve and photospheric velocity of the SN are well converged in the time domain of interest. We tried following two possible powering mechanisms for SN 2015ap using SNEC, as follows.

\subsubsection{Ni-Co Decay}

The radioactive decay of $\mathrm{Ni}$ and $\mathrm{Co}$ is considered to be the most prominent mechanism for the powering of light curves of SNe Ib (e.g., Karamehmetoglu 2017). SNEC incorporates this model by default. Here, we provide the setup of the explosion parameters to incorporate the $\mathrm{Ni}-\mathrm{Co}$ decay model. The code does not include a nuclear-reaction network, and ${ }^{56} \mathrm{Ni}$ is given by hand. We considered two scenarios of nickel distribution. In one case, the mass of $\mathrm{Ni}$ is set to be $M_{\mathrm{Ni}}=0.135 M_{\odot}$ and distributed from the inner 


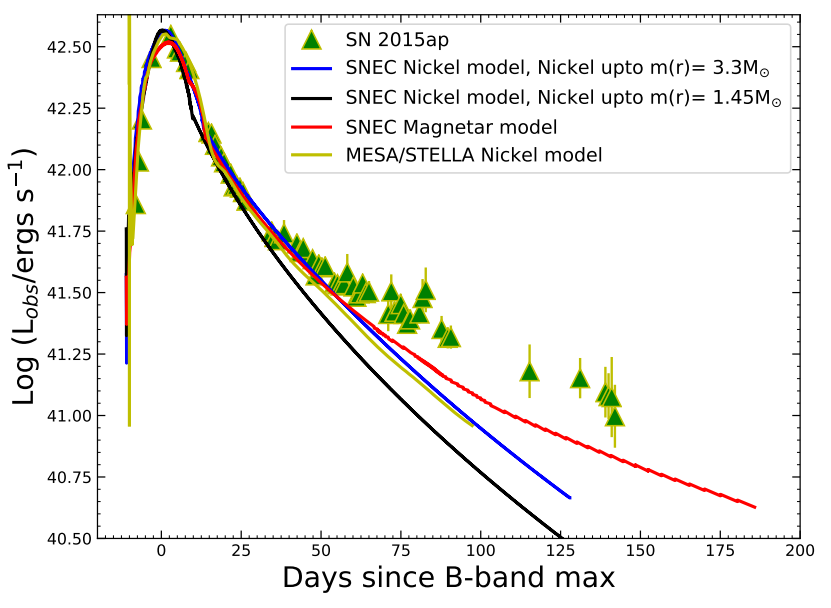

Figure 21. Comparison of quasi-bolometric light curve of SN 2015ap with those obtained using SNEC by taking into account the Ni and Co decay model and the magnetar model. This figure also depicts the result of the $\mathrm{Ni}-\mathrm{Co}$ decay model obtained using STELLA.

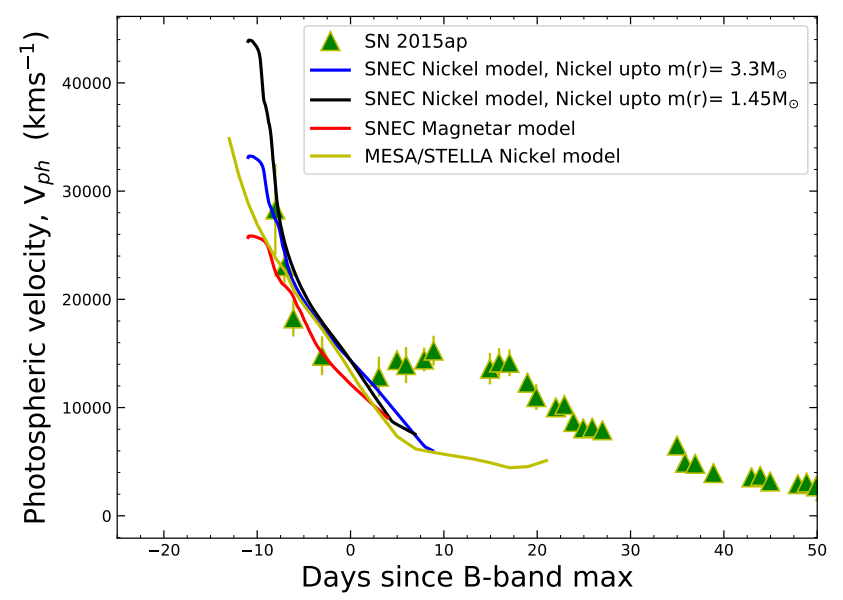

Figure 22. Comparison of the observed velocity evolution of SN 2015ap with that produced by SNEC using two models. We also show a comparison of the observed velocity evolution produced with STELLA.

boundary up to the mass coordinate $m(r)=3.3 M_{\odot}$. For this model the explosion is simulated as a piston, with the first two computational cells of the profile boosted outward with a velocity of $4.0 \times 10^{9} \mathrm{~cm} \mathrm{~s}^{-1}$ for a time interval of $0.01 \mathrm{~s}$. The total energy $\left(E_{\text {tot }}\right)$ of the model is $3.7 \times 10^{51} \mathrm{erg}$. For the second case, the mass of $\mathrm{Ni}$ is set to be $M_{\mathrm{Ni}}=0.1 M_{\odot}$ and distributed near the centre, from the inner boundary up to the mass coordinate of $m(r)=1.45 M_{\odot}$. The explosion is simulated as a piston, with the first two computational cells of the profile boosted outward with a velocity of $5.0 \times 10^{9} \mathrm{~cm} \mathrm{~s}^{-1}$ for a time interval of $0.01 \mathrm{~s}$. The total energy of the model in this case is $6.5 \times 10^{51} \mathrm{erg}$. Thus, our results provide a range of $M_{\mathrm{Ni}}$ and total energy, depending on the distribution of nickel mass.

\subsubsection{Spin-down of a magnetar}

We also tried a magnetar-powering mechanism for the light curve as SN 2015ap; it shows some resemblance to SN 2008D, which has broader features in its early-time spectra and also some X-ray emission in the later phases. A few SNe Ib are also explained by magnetar models, one such example being SN 2005bf (Maeda et al. 2007). For this model, the explosion is the piston type, with the first two computational cells of the profile boosted outward with a velocity of $3.3 \times 10^{9} \mathrm{~cm} \mathrm{~s}^{-1}$ for a time interval of $0.01 \mathrm{~s}$. The total energy of the model in this case is $2.2 \times 10^{51} \mathrm{erg}$. The most important change made to Ouchi \& Maeda (2019) is that we add the magnetar heat to the ejecta.

Following Metzger et al. (2015), the magnetar spindown luminosity is given by

$L_{\mathrm{sd}}=L_{\mathrm{sd}_{\mathrm{i}}}\left(1+t / t_{\mathrm{sd}}\right)^{-2}$.

Here, $L_{\mathrm{sd}_{\mathrm{i}}}$ is the spin-down luminosity at $t=0$, and $t_{\mathrm{sd}}$ is the initial spin-down time. We inject this luminosity into the whole ejecta above the mass cut uniformly in mass. For the initial spin-down luminosity, we assume $L_{\mathrm{sd}_{\mathrm{i}}}=1.3 \times$ $10^{43} \mathrm{erg} \mathrm{s}^{-1}$, while for the initial spin-down time, we assume $t_{\mathrm{sd}}=12 \mathrm{~d}$. In this model, we do not include the effect of $\mathrm{Ni}$ heating. Following Metzger et al. (2015) (Equations 2 and $3)$, corresponding to $L_{\mathrm{sd}_{\mathrm{i}}}=1.3 \times 10^{43} \mathrm{erg} \mathrm{s}^{-1}$ and $t_{\mathrm{sd}}=12 \mathrm{~d}$, we obtain a magnetic field $(B)$ of $5.1 \times 10^{14} \mathrm{G}$ and an initial period $\left(P_{i}\right)$ of $43.06 \mathrm{~ms}$ for the modelled magnetar. These values of $B$ and $P_{i}$ are very close to those obtained using MINIM.

We also use the public version of STELLA, available with MESA. The default radioactive decay of ${ }^{56} \mathrm{Ni}$ and ${ }^{56} \mathrm{Co}$ as a powering mechanism is used for SN 2015ap. Nearly similar parameters as in the case of SNEC are used for STELLA. The MESA setups are unchanged for STELLA calculations. We use a total energy after explosion of $3.6 \times 10^{51}$ erg and a ${ }^{56} \mathrm{Ni}$ mass of $0.193 M_{\odot}$, which is slightly more than the ${ }^{56} \mathrm{Ni}$ mass used in SNEC. In order to avoid the numerical problem caused by the high-velocity material, we removed the outer layer of the progenitor where the density is less than $10^{-5} \mathrm{~g} \mathrm{~cm}^{-3}$ (see also Moriya et al. 2020).

\subsubsection{Summary of the modelling}

Figure 21 shows the comparison of the observed quasibolometric luminosity with that produced by SNEC. We find that the radioactive decay models with ${ }^{56} \mathrm{Ni}$ mass in the range $0.1-0.135 M_{\odot}$ and total energy in the range (3.7$6.5) \times 10^{51}$ erg could nicely explain the light curve. Our results also signify that the distribution of ${ }^{56} \mathrm{Ni}$ mass plays an important role for explaining the observed light curves. The magnetar model could also explain the quasi-bolometric light curve, but we do not see very strong evidence that SN 2015ap is powered by a magnetar. This figure also shows the results of STELLA calculations. We see that, although the explosion energy is similar, we need a slightly higher amount of nickel to properly match the observed light curve of SN 2015ap.

Figure 22 illustrates a comparison of observed photospheric velocity evolution produced by blackbody fitting with that produced by SNEC. We see that the $\mathrm{Ni}-\mathrm{Co}$ decay and magnetar models initially show higher velocities, 
but in the later epochs they well replicate the observed photospheric velocities. This figure also shows the Fe II $5169 \AA$ velocity evolution produced by STELLA. Owing to the unambiguous absence of Fe II $5169 \AA$ features in SN 2015ap, we used the photospheric velocity obtained through a blackbody fit for our comparison of Fe II $5169 \AA$ line velocities. We can see a very good match between the STELLA velocities and observed ones. The modelling parameters, along with the observed values, are listed in Table 3.

\subsection{SN 2016bau}

Using a similar progenitor model on the verge of corecollapse, obtained through MESA, as in the case of SN 2015ap, we carried out the radiation hydrodynamical simulations using SNEC and STELLA. For calculations using SNEC, the setup closely follows that of Ouchi \& Maeda (2019). First, we excise the innermost $1.53 M_{\odot}$ before the explosion, assuming that it collapses to form a neutron star. The number of cells is set to 70 . We try two possible powering mechanisms for SN 2016bau using SNEC, as follows.

\subsubsection{Ni-Co Decay}

The setup for the calculation using SNEC is similar to that of SN 2015ap. Considering the radioactive decay of $\mathrm{Ni}-\mathrm{Co}$ the most prominent mechanism for powering light curves of $\mathrm{SNe} \mathrm{Ib}$, we employed this model as the powering mechanism for SN 2016bau. Here we briefly describe the setup of the explosion parameters incorporated in the $\mathrm{Ni}-\mathrm{Co}$ decay model.

We considered two cases of ${ }^{56} \mathrm{Ni}$ mass distribution. In the first case, the mass of ${ }^{56} \mathrm{Ni}$ synthesised is set to be $M_{\mathrm{Ni}}=0.045 M_{\odot}$. Then, it is distributed from the inner boundary up to the mass coordinate of $M(r)=3.3 M_{\odot}$. Thereafter, we simulate the explosion as a piston, with the first two computational cells of the profile boosted outward with a velocity of $4.2 \times 10^{9} \mathrm{~cm} \mathrm{~s}^{-1}$ for a time interval of $0.01 \mathrm{~s}$. The model has a total energy of $1.3 \times 10^{51} \mathrm{erg}$. For the second case, the mass of ${ }^{56} \mathrm{Ni}$ synthesised is set to be $M_{\mathrm{Ni}}=0.03 M_{\odot}$ and distributed from the inner boundary up to the mass coordinate of $M(r)=1.57 M_{\odot}$. Thereafter, we simulate the explosion as a piston, with the first two computational cells of the profile boosted outward with a velocity of $4.9 \times 10^{9} \mathrm{~cm} \mathrm{~s}^{-1}$ for a time interval of $0.01 \mathrm{~s}$, and the model has a total energy of $1.93 \times 10^{51} \mathrm{erg}$. We find that, depending on the $M_{\mathrm{Ni}}$ distribution, the radioactive decay models with ${ }^{56} \mathrm{Ni}$ mass in the range $0.03-0.045 M_{\odot}$ and total energy in the range $(1.23-1.93) \times 10^{51}$ erg can nicely explain the light curve.

\subsubsection{Spin-down of a magnetar}

We also tried the magnetar powering mechanism for SN 2016bau. The explosion is simulated as a piston, with the first two computational cells of the profile boosted outward with a velocity of $3.6 \times 10^{9} \mathrm{~cm} \mathrm{~s}^{-1}$ for a duration of $0.01 \mathrm{~s}$ and a total model energy of $0.8 \times 10^{51} \mathrm{erg}$. Like SN 2015ap, we inject $L_{\mathrm{sd}_{\mathrm{i}}}=0.4 \times 10^{43} \mathrm{erg} \mathrm{s}^{-1}$ to the ejecta above the mass cut uniformly in mass. We assume $t_{\mathrm{sd}}=16.0 \mathrm{~d}$ and do not include the effect of $\mathrm{Ni}$ heating.

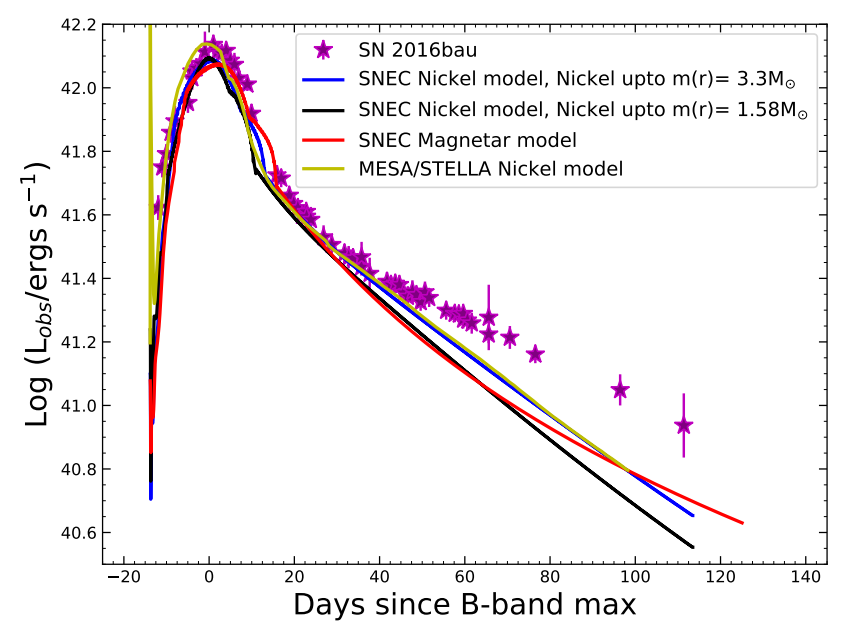

Figure 23. Comparison of the quasi-bolometric light curve of SN 2016bau and light curves produced by SNEC and STELLA, considering the radioactive decay model.

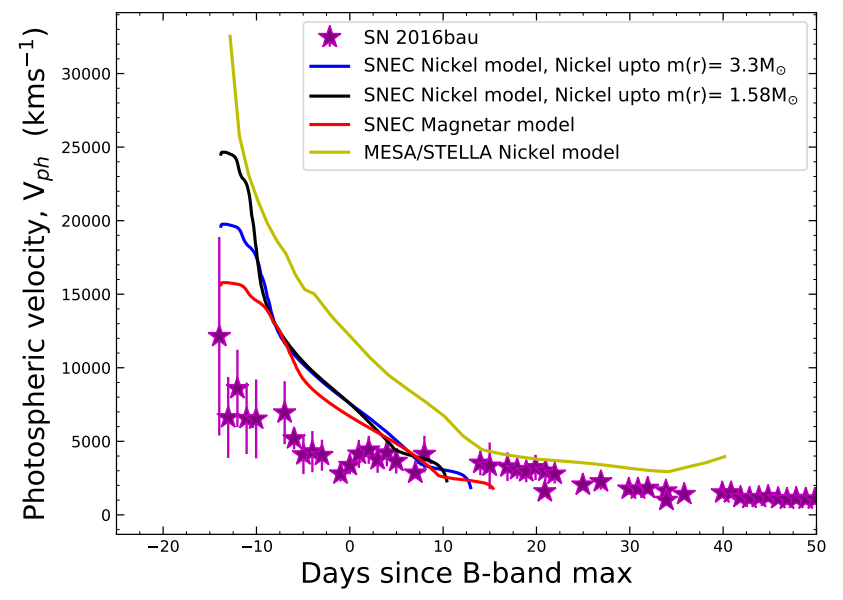

Figure 24. Comparison between the observed velocity evolution of SN 2016bau and the photometric velocity evolution produced by SNEC and STELLA, considering the radioactive decay model.

Corresponding to $L_{\mathrm{sd}_{\mathrm{i}}}=0.4 \times 10^{43} \mathrm{erg} \mathrm{s}^{-1}$ and $t_{\mathrm{sd}}=16 \mathrm{~d}$, we obtain a magnetic field $(B)$ of $7.9 \times 10^{14} \mathrm{G}$ and an initial period $\left(P_{i}\right)$ of $71.8 \mathrm{~ms}$ for the modelled magnetar. These values of $B$ and $P_{i}$ are very close to those obtained using MINIM.

We also perform STELLA calculations for SN 2016bau, by employing the default radioactive decay of ${ }^{56} \mathrm{Ni}$ and ${ }^{56} \mathrm{Co}$ powering mechanism. We used similar parameters as in the case of SNEC and STELLA. The MESA setups are unchanged for STELLA calculations. We used a total energy after explosion of $1.6 \times 10^{51} \mathrm{erg}$ and a ${ }^{56} \mathrm{Ni}$ mass of $0.065 M_{\odot}$, slightly above the ${ }^{56} \mathrm{Ni}$ mass used in SNEC.

\subsubsection{Summary of the modelling}

Figure 23 shows the comparison of the observed quasibolometric luminosity with that produced by SNEC for the radioactive decay and magnetar spin-down models. We find 
Table 3. Observed and modelled parameters for SN 2015ap and SN 2016bau

\begin{tabular}{cccc}
\hline \hline & $\begin{array}{c}M_{\mathrm{Ni}} \\
\left(M_{\odot}\right)\end{array}$ & $\begin{array}{c}M_{\mathrm{ej}} \\
\left(M_{\odot}\right)\end{array}$ & $\begin{array}{c}E_{\mathrm{tot}} \\
10^{51} \mathrm{erg}\end{array}$ \\
\hline \hline SN 2015ap & & & \\
\hline Arnett's model & & \\
\hline SNEC (Ni distributed up to $\left.M(r)=3.3 M_{\odot}\right)$ & $0.14 \pm 0.02$ & $2.2 \pm 0.6$ & $*$ \\
SNEC (Ni distributed up to $\left.M(r)=1.45 M_{\odot}\right)$ & 0.135 & 2.02 & 3.7 \\
SNEC Magnetar model & 0.1 & 2.02 & 6.5 \\
From STELLA & 0.0 & 2.02 & 2.2 \\
SN 2016bau & 0.193 & 1.92 & 3.6 \\
\hline Arnett's model & & & \\
\hline SNEC (Ni distributed up to $\left.M(r)=3.3 M_{\odot}\right)$ & $0.055 \pm 0.006$ & $1.6 \pm 0.3$ & $* *$ \\
SNEC (Ni distributed up to $\left.M(r)=1.57 M_{\odot}\right)$ & 0.045 & 1.89 & 1.23 \\
SNEC Magnetar model & 0.03 & 1.89 & 1.93 \\
From STELLA & 0.0 & 1.89 & 0.8 \\
\hline \hline
\end{tabular}

$a$ : Calculated using $t_{\text {rise }}, \kappa=0.07 \mathrm{~cm}^{2} \mathrm{~g}^{-1}$, and $L_{\text {peak }}$

$*$ : Instead, a kinetic energy of the ejecta $\left(E_{k}\right)=1.05 \times 10^{51} \mathrm{erg}$ is obtained.

$* *$ : Instead, a kinetic energy of the ejecta $\left(E_{k}\right)=0.24 \times 10^{51} \mathrm{erg}$ is obtained.

that the radioactive decay model from SNEC could successfully explain the light curve. The magnetar model also shows a good match, but we do not see any significant signs of SN 2016bau being powered by a magnetar. This figure also shows the results of STELLA calculations; they also match the observed light curve of SN 2016bau nicely, with parameters similar to those of SNEC.

Figure 24 shows a comparison of the observed photospheric velocity evolution produced by blackbody fitting with that produced by the radioactive decay model and the magnetar spin-down model using SNEC. Here, the models show high initial velocities which drop at later epochs. We see that the velocities produced by the models initially deviate from the observed photospheric velocities, but tend to follow velocities similar to the observed ones at later epochs. The figure also shows a comparison the Fe II $5169 \AA$ line velocity obtained using STELLA with the photospheric velocity obtained through blackbody fits. Similar to the case of SN 2015ap, this SN also lacks unambiguous features of the Fe II $5169 \AA$ line.

From Figure 24, it is evident that our model overestimates the velocity by a factor of nearly two. The $M_{\mathrm{ej}}$ from Arnett's model is $\sim 1.6 M_{\odot}$. The diffusion time is given by $\left(M^{3} / E\right)^{1 / 4}$. Since this combination is fixed, $M^{3} / E$ is roughly constant. But $M^{3} / E \approx M^{3} /\left(M v^{2}\right)=M^{2} / v^{2} \approx$ constant. Thus, we have $M \approx v$ to meet the observational constraints. So, if we need to decrease the velocity by a factor of two, the ejecta mass would also decrease by the same factor. Then the He star mass is likely $\sim 2-2.5 M_{\odot}$, which is at the boundary between a SN and a non-SN. Since $E \approx M v^{2} \approx v^{3}$, the energy may go down quite substantially in this case.

\section{DISCUSSION}

We present a detailed photometric and spectroscopic analysis of two Type Ib SNe, namely SN 2015ap and SN 2016bau. From our analysis, SN 2015ap is an intermediate-luminosity normal SN Ib, while SN 2016bau is highly extinguished by host-galaxy dust. In this section, we discuss the major outcomes of our present analysis.

The photometric properties of both the SNe were analysed by determining the bolometric luminosity of their light curves. For SN 2015ap, we calculate a ${ }^{56} \mathrm{Ni}$ mass of $0.14 \pm 0.02 M_{\odot}$ and an ejecta mass of $2.2 \pm 0.6 M_{\odot}$, while for SN 2016bau, the ${ }^{56} \mathrm{Ni}$ mass and ejecta mass were $0.055 \pm 0.006 M_{\odot}$ and $1.6 \pm 0.3 M_{\odot}$, respectively. The photospheric temperature, radius, and velocity evolution for both $\mathrm{SNe}$ were also explored. Based on the derived physical quantities from the temporal evolution of these two SNe, we tried to constrain possible powering mechanisms using a semi-analytical model called MINIM. We found that the semianalytical RD model failed to simultaneously fit the early and late phases of both $\mathrm{SNe}$, raising issues related to the validity of such models in the case of stripped-envelope SNe. One solution to such a situation is to fit the early-phase and late-phase data with different sets of model parameters. Another cause for the failure of the RD model can be attributed to the assumption of the constant ejecta density profile. For the MAG model, the fitted parameters seemed to be unphysical in both $\mathrm{SNe}$, especially the very slow initial rotation (for SN 2015ap, $P_{i} \approx 40 \mathrm{~ms}$, and for SN 2016bau, $P_{i} \approx 70 \mathrm{~ms}$ ) and the very low initial rotational energy (for both $\mathrm{SNe}$, $E_{p} \approx 10^{49} \mathrm{erg}$ ). Also, no signs of these SNe powered by a magnetar mechanism were evident either from photometry or spectroscopy, so this possibility was discarded.

The spectroscopic behaviour of both SNe was also studied using the present and archival data. These SNe showed unambiguous He I features from very early to late phases, confirming them to be SNe Ib. Their spectral features match with those of other well-studied SNe Ib. Additionally, SN 2015ap closely resembled SN 2008D, which had shown X-ray emission. The spectra of our two SNe at various epochs were modelled using SYN++. For SN 2015ap, the spectral modelling indicated a range of photospheric temperatures and velocities from $13,000 \mathrm{~km} \mathrm{~s}^{-1}$ to $6800 \mathrm{~km} \mathrm{~s}^{-1}$ and from $12,000 \mathrm{~K}$ to $4500 \mathrm{~K}$ (respectively) during the time interval $-7 \mathrm{~d}$ to $+33 \mathrm{~d}$. For SN $2016 \mathrm{bau}$, these two parameters ranged from $16,000 \mathrm{~km} \mathrm{~s}^{-1}$ to $8000 \mathrm{~km} \mathrm{~s}^{-1}$ and from $9000 \mathrm{~K}$ to $4000 \mathrm{~K}$ (respectively) during the time interval $-14 \mathrm{~d}$ to $+33 \mathrm{~d}$. The spectra of these two SNe at particular epochs were compared with model spectra of 12,13 , and $17 M_{\odot}$ progenitor stars. For SN 2015ap, the $12 M_{\odot}$ and 17 $M_{\odot}$ model spectra showed reasonable matches, indicating a progenitor in the mass range $12-17 M_{\odot}$; for SN 2016bau, the $12 \mathrm{M}_{\odot}$ model spectrum could explain the observed spectrum to some extent better than other models, indicating a $\leq 12 M_{\odot}$ progenitor. 
Based on the photometric and spectroscopic properties described above, a $12 M_{\odot}$ ZAMS star was chosen as the possible progenitor for both SNe. This $12 M_{\odot}$ ZAMS progenitor was evolved up to the onset of core collapse using MESA. The MESA outputs on the onset of core collapse were fed as input to SNEC and STELLA, which simulate the synthetic explosions. The RD and MAG models were employed using SNEC while only the RD model was employed in STELLA. Here also, the models failed to fit the late part of the light curve simultaneously. The cause can be attributed to the various assumptions, including the spherically symmetrical explosions and the use of constant ejecta density profiles throughout. Similar to the case of MINIM, the MAG model provided unphysical parameters during the SNEC analysis.

Our analysis favours a $12 M_{\odot}$ ZAMS star as a possible progenitor for SN 2015ap based on outputs of the models reasonably explaining the bolometric luminosity light curve and the photospheric velocity. However, in case of SN 2016bau, the model velocities are higher by a factor of almost 2, demanding that the ejecta mass be lower by similar factor. This would imply an He star of mass $\sim 2-2.5 M_{\odot}$, which is at the boundary for exploding as an SN. Thus, a slightly lower mass ZAMS star could also be the possible progenitor of SN 2016bau. Hydrodynamical evolution models of such low-mass stars to reach the stage of core collapse and then undergo synthetic explosions are extremely difficult to perform, but this can be taken as a challenge for the future.

\section{CONCLUSIONS}

Photometric and spectroscopic analyses of the Lick/KAITdiscovered Type Ib SN 2015ap and another Type Ib SN 2016bau, both having extensive follow-up observations made with various telescopes, are discussed. For both SNe, photometric data corrected for the Milky Way and hostgalaxy extinction were used to estimate the quasi-bolometric luminosity light curves and study the photospheric radius, temperature, and velocity evolution. Spectral properties of SN 2015ap and SN 2016bau were then explored in detail. We modelled the spectra, studied the spectral evolution, and compared the spectra at various epochs with those of other well-studied SNe Ib, which further confirmed that these two SNe are Type Ib SN.

We attempted to determine the progenitor masses of SN 2015ap and SN 2016bau. Our results support $12 M_{\odot}$ progenitors for these two SNe. The $12 M_{\odot}$ ZAMS progenitor was evolved up to the onset of core-collapse using MESA. The output of MESA was incorporated as input to SNEC and STELLA, which produced the artificial explosions replicating the actual SN explosions. Considering the decay of $\mathrm{Ni}$ and Co to be the most prominent powering mechanism for $\mathrm{SNe} \mathrm{Ib}$, we tried this powering mechanism for SN 2015ap and SN 2016bau, using SNEC and STELLA. We found that the quasi-bolometric luminosity could nicely be explained by our models, while the velocity evolution obtained from SNEC and STELLA satisfactorily agrees with the observed one. We also explored the effect of the distribution of nickel mass near the centre and up to near the surface. Lower amounts of nickel were required to match the light curve for the case of centrally distributed nickel in comparison to the case where the nickel was distributed up to near the surface. Based on the above conclusions, our analysis supports a star having $M_{\mathrm{ZAMS}}=12 M_{\odot}$ as the possible progenitor for SN 2015ap. For SN 2016bau, a slightly lower ZAMS progenitor is expected.

\section{ACKNOWLEDGEMENTS}

We are highly thankful to the anonymous referee for providing a very helpful report. We acknowledge Sanyum Channa, Maxime de Kouchkovsky, Andrew Halle, Michael Hyland, Minkyu Kim, Kevin Hayakawa, Kyle McAllister, Jeffrey Molloy, Andrew Rikhter, Benjamin Stahl, and Yinan Zhu for obtaining some of the Lick observations. We also thank the MESA troubleshooting team, especially Jared Goldberg, for constant guidance. We also acknowledge Van Dyk Schuyler for useful discussion regarding the manuscript. A.A., S.B.P., R.G., and K.M. acknowledge BRICS grant DST/IMRCD/BRICS/Pilotcall/ProFCheap/2017(G). A.A. also acknowledges funds and assistance provided by the Council of Scientific \& Industrial Research (CSIR), India. R.O. acknowledges support provided by Japan Society for the Promotion of Science (JSPS) through KAKENHI grant (19J14158). K.M. acknowledges support provided by the Japan Society for the Promotion of Science (JSPS) through KAKENHI grants JP17H02864, JP18H04585, JP18H05223, JP20H00174, and JP20H04737. Support for A.V.F.'s supernova research group has been provided by the TABASGO Foundation, the Christopher R. Redlich Fund, and the U.C. Berkeley Miller Institute for Basic Research in Science (where A.V.F. is a Senior Miller Fellow). Additional support was provide by NASA/HST grant GO-15166 from the Space Telescope Science Institute (STScI), which is operated by the Associated Universities for Research in Astronomy, Inc. (AURA), under NASA contract NAS 5-26555. J.V. is supported by the project "Transient Astrophysical Objects" (GINOP 2.3.2-15-2016-00033) of the National Research, Development, and Innovation Office (NKFIH), Hungary, funded by the European Union.

Lick/KAIT and its ongoing operation were made possible by donations from Sun Microsystems, Inc., the HewlettPackard Company, AutoScope Corporation, Lick Observatory, the U.S. National Science Foundation, the University of California, the Sylvia \& Jim Katzman Foundation, and the TABASGO Foundation. Research at Lick Observatory is partially supported by a generous gift from Google. Some of the data presented herein were obtained at the W. M. Keck Observatory, which is operated as a scientific partnership among the California Institute of Technology, the University of California, and NASA; the observatory was made possible by the generous financial support of the W. M. Keck Foundation. The Lick and Keck Observatory staff provided excellent assistance with the observations.

\section{DATA AVAILABILITY}

The photometric and spectroscopic data used in this work can be made available on request to the corresponding author. The inlist files to create the MESA models and STELLA 
calculations, along with SNEC parameters files, can also be made available on request to the corresponding author.

\section{REFERENCES}

Arnett W. D. 1980, ApJ, 237, 541

Arnett W. D. 1982, ApJ, 253, 785

Arnett W. D.\& Fu, A. 1989, ApJ, 340, 396

Arnett D., 1996, Supernovae and Nucleosynthesis,ed. D. N. Spergel, Princeton series in astrophysics, Princeton,NJ; Princeton University Press

Ann H. B., Seo M., \& Ha D. K. 2015, ApJS, 217, 27A

Arbour, R. 2016, TNSTR, 215, 1

Bessell, M. S., Castelli, F., Plez, B. 1998, A\&A, 333, 231

Bersten, M. C., Benvenuto, O. G., Nomoto, K., Ergon, M., Folatelli, G. et al. 2012, ApJ, 757, 31

Bersten, M. C., Benvenuto, O. G., Folatelli, G., Nomoto, K., Kuncarayakt H. et al. 2014, AJ, 148, 68

Bersten, M. C., Folatelli, G., García, F., van Dyk, S. D., Benvenuto, O. G. et al. 2018, Nature, 554, 497

Blinnikov S. I., Eastman R., Bartunov O. S., Popolitov V. A., \& Woosley S. E. 1998, ApJ, 496, 454

Blinnikov S., Lundqvist P., Bartunov O., Nomoto K., \& Iwamoto K. 2000, ApJ, 532, 1132

Blinnikov S. I., Röpke F. K., Sorokina E. I. et al. 2006, A\&A, 453, 229

Branch D., benetti S., Kasen Daniel, Baron E., Jeffery D. J. et al. 2002, ApJ, 566, 1005

Branch, D., Jeffery, D. J., Young, T. R., \& Baron, E. 2006, PASP, 118, 791

Brachetti P., de Felice Ciccoli M., di Pillo G. et al. 1997, J. Global Optimization, 10, 165

Branch D., Parrent J., Troxel M. A., Casebeer D., Jeffery D. J. et al. 2007, American Institute of Physics Conference Series Vol. 924, The Multicolored Landscape of Compact Objects and Their Explosive Origins. pp 342-349, doi:10.1063/1.2774879

Brown, Peter J.; Breeveld, A. A., Holland, S., Kuin, P., Pritchard, T. 2014, Astrophysics and Space Science, 354(1), 89-96

Cao Y., Kasliwal M. M., Arcavi I. et al. 2013, ApJ, 775, L7

Chatzopoulous, E., Wheeler, J. C., Vinko, J., Horvath, Z. L., \& Nahy, A. 2013, ApJ, 773, 76

Chevalier R. A. 1982, ApJ, 258, 790

Chevalier R. A., \& Fransson C. 1994, ApJ, 420, 268

Chengalur, J., Salpeter, E. \& Terzlan, Y. 1993, ApJ, 419, 30

Crockett R. M. et al. 2008, MNRAS, 391, L5

de Jager, C., Nieuwenhuijzen, H., \& van der Hucht, K. A. 1988, A\&AS, 72, 259

Dessart L., Hillier D. J., Li, C., et al. 2012, MNRAS, 424, 2139

de Vaucouleurs G., de Vaucouleurs A., Corwin H. G., Jr., Buta R. J., Paturel G., \& Fouqué, P. 1991, RC3.9, "Third Reference Catalogue of Bright Galaxies," version 3.9 (New York: Springer)

Eldridge, J. J., \& Langer, N., Tout, C. A. 2011, MNRAS, 414, 3501

Eldridge, J. J., \& Maund, J. R. 2016, MNRAS, 461, L117

Elmhamdi A., Danziger I. J., Branch D., Leibundgut B., Baron E. et al. 2006, A\&A, 450, 305

Falco E. E., Kurtz M. J., Geller M. J., Huchra J. P., Peters J. et al. 1999, PASP, 111, 438

Filippenko, A. V. 1988, AJ, 96, 1941

Filippenko, A. V., Matheson T., Ho L. C. 1993, ApJL, 481, L89

Filippenko, A. V. 1997, ARA\&A, 35, 309

Filippenko, A. V., Li, W. D., Treffers, R. R., \& Modjaz, M. 2001, in Small-Telescope Astronomy on Global Scales., ed. B.Paczyński, W. P. Chen, \& C. Lemme (San Francisco: ASP), 121

Folatelli G. et al. 2014, ApJ, 793, L22
Fukugita, M., Ichikawa, T., Gunn, J. E., Doi, M.,Shimasaku, K. et al. 1996, AJ, 111, 1748

Gal-Yam, A. 2017, in Handbook of Supernovae (Springer International Publishing), 1

Gangopadhyay A., Misra K., Sahu D. K., Wang, Shan-Qin, Kumar B. et al. 2020, MNRAS, 497, 3770

Garry., G. 2004, Science, Vol. 304, Issue 5679, pp. 1915-1916, DOI: $10.1126 /$ science. 1100370

Gaskell C. M., Cappellaro E., Dinerstein H. L.,Garnett D. R., Harkness R. P., Wheeler J. C. 1986, ApJ, 306 L77

Ginzberg, S., \& Balberg, S. 2012, ApJ, 757178

Groh, J. H., Maynet, G., Georgy, C., \&, Ekstrom, S. 2013, A\&A, 558, A131

Groh Jose H. 2017, Predicting the nature of supernova progenitors, Phil. Trans. R. Soc. A., 375, 20170219 (https://doi.org/10.1098/rsta.2017.0219)

Guillochon, J., Nicholl, M., Villar, V. A., Mockler, B., Narayan, G. et al. 2018, The Astrophysical Journal Supplement Series, 236:6

Hachinger, S., Mazzali, P. A., Taubenberger, S., et al. 2012,MNRAS, 422,70

Henyey, L., Vardya, M. S., \& Bodenheimer, P. 1965, ApJ, 142, 841

Herwig, F. 2000, A\&A, 360, 952

Jerkstrand, A., Ergon, M., Smartt, S. J., Fransson, C., Sollerman, J. 2015, A\&A, 573, A12

Kasen, D., \& Bildsten, L. 2010, ApJ, 717, 245

Karamehmetoglu, E., Taddia, F., Sollerman, J., Wyrzykowski, Ł., Schmidl, S. et al. 2017, A\&A, 602, A93

Kippenhahn, R., Ruschenplatt, G., \& Thomas, H.-C. 1980, A\&A, 91, 175

Kumar, A., Pandey S. B., Konyves-Toth, R., et al. 2020, ApJ, $892,28 \mathrm{~K}$

Kumar, A., Kumar, B., Pandey, S. B., et al. 2021, MNRAS, 502, 1678

Landolt, A. 1992, AJ, 104, 340

Langer, N., El Eid, M. F., \& Fricke, K. J. 1985, A\&A, 145, 179

Li W., Filippenko A. V., Chornock R. \& Jha S., et al. 2003, PASP, 115,844

Maeda, K., Mazzali, P. A., Deng, J., Nomoto, K., Yoshii, Y. et al. 2003, ApJ, 593, 931

Maeda, K., Tanaka, M., Nomoto, K., Tominaga, N., Kawabata, K. et al. 2007, ApJ, 666, 1069

Metzger, B. D., Margalit, B., Kasen, D., et al. 2015, MNRAS, 454,3311

Milisavljevic, D., Fesen R. A., Gerardy, C. L., et al. 2014, AJ, 147,99

Miller, J. S., \& Stone, R. P. S. 1993, Lick Obs. Tech. Rep. No. 66

Modjaz, M., Blondin, S., Kirshner, R. P. et al. 2014, AJ, 147, 99

Moriya, T. J., Tominaga, N., Blinnikov, S. I., Balkanov, P. V., \& Sorokina, E. I. 2011, MNRAS, 415, 199

Moriya, T. J., Suzuki, A., Takiwaki, T., et al. 2020, MNRAS, 497, 1619

Morozova, V., Piro, A. L., Renzo, M., Ott, C. D. 2015, ApJ, 814, 63

Nadyozhin, D. K. 1994, ApJS, 92, 527

Nicholl, M., Guillochon, J., \& Berger, E. 2017, ApJ, 850, 55

Nicholl, M. 2018, RNAAS, 2, 230

Nomoto K., I., Iwamoto, K., Suzuki, T. 1995, Phys. Rep., 256, 173

Nugis, T., \& Lamers, H. J. G. L. M. 2000, A\&A, 360, 227

Oke, J. B., Cohen J. G., Carr M., Cromer J., Dingizian A., et al. 1995, PASP, 107, 375O

Ostriker, J. P., \& Gunn, J. E. 1971, ApJL, 164, L95

Ouchi, R., \& Maeda, K. 2017, ApJ, 840, 90

Ouchi, R., \& Maeda, K. 2019, ApJ, 877, 92

Pandey S. B. et al. 2020, In preparation 
Paxton, B., Bildsten, L., Dotter, A., Herwig, F., Lesaffre, P. et al. 2011, ApJS, 192, 3

Paxton, B., Cantiello, M., Arras, P., Bildsten, L., Brown, E. F. et al. 2013, ApJS, 208, 4

Paxton, B., Marchant, P., Schwab, J., Bauer, E. B., Bildsten, L. et al. 2015, ApJS, 220, 15

Paxton, B., Schwab, J., Bauer, E. B., Bildsten, L., Blinnikov, S. et al. 2018, ApJS, 234, 34

Podsiadlowski P., Joss P. C., Hsu J. J. L. 1992, ApJ, 391, 246

Prentice S. J., Mazzali, P., Pian E., Gal-Yam A., Kulkarni S. R. et al. 2016, MNRAS, 458, 2973

Prentice S. J., Ashall C., James P. A., et al. 2019, MNRAS, 485, 15592973

Richardson D., Jenkins R. L. III, Wright J.,\& Maddox, L. 2014, AJ, 147, 118

Ross, W., Zheng, W. \& Filippenko, A. 2015, CBET, 4190

Sahu, D. K.,Gurugubelli U. K., Anupama G. C., Nomoto K. 2011, MNRAS, 413, 2583

Schlafly, Edward F., \& Finkbeiner, Douglas P. 2011, ApJ, 737, 103

Sher, D. 1968, JRASC, 62, 105S

Shivvers I., Filippenko A. V., Silverman J. M., Zheng W., Foley R. J. et al. 2018, MNRAS, 482, 1545

Smartt S. J. 2009, ARA\&A, 47, 63

Smartt, S. J. 2015, PASA, 32, e016

Srivastav, S., Anupama G. C., Sahu D. K. 2014a, MNRAS, 445, 1932

Stahl B. E., Zheng W., de J. Thomas, Filippenko A. V., Bigley, A. et al. 2019, MNRAS 490, 3882

Stetson, P. B. 1987, PASP, 99, 191

Stritzinger, M., Mazzali, P., Phillips, M. M., Immler, S., Soderberg, A. et al. 2009, APJ, 696, 713

Stritzinger M. D., Taddia F., Burns C. R., Phillips M. M., Bersten M. et al. 2018, A\&A, 609, A135

Tartaglia, L., Elias-Rosa, N., Benetti, S., Cappellaro, E., Tomasella, L., P. Ochner, A. Pastorello, G. et al., The Astronomer's Telegram, No.8039,2015

Thomas, R. C., Nugent, P. E., \& Meza, J. C., 2011, PASP, 123, 237

Tonry, J. L., Stubbs, C. W., Lykke, K. R., et al., 2012, ApJ, 750, 99

Uomoto, A. 1986, ApJL, 310, L35

Valenti, S., Elias-Rosa, N., Taubenberger, S et al., 2008, ApJL, $673, \mathrm{~L} 155$

Van Dyk, S. D., Zheng, W., Clubb, K. I., Filippenko, A. V., Cenko, S. B. et al. 2013, ApJL, 772, L32

Vink, J. S., de Koter, A., \& Lamers, H. J. G. L. M. 2001, A\&A, 369,574

Wheeler, J. C., Johnson, V. \& Clocchiatti, A., 2015, MNRAS, 450, 1295

Wheeler, J. C., Chatzopoulos, E., Vinkó, J., et al. 2017, ApJ, 851, L14

Woosley, S. E., \& Janka, T. 2005, Nature Physics, 1, 147

Woosley, S. E. 2010, ApJL, 719, L204

Yoon, S.-C., Woosley, S. E., \& Langer, N. 2010, ApJ, 725, 940

Yaron, O., \& Gel-Yam, A. 2012, PASP, 124, 668

\section{APPENDIX A: LOG OF SPECTROSCOPIC AND PHOTOMETRIC OBSERVATIONS}

This paper has been typeset from a $\mathrm{T}_{\mathrm{E}} \mathrm{X} / \mathrm{LATEX}$ file prepared by the author. 
Table A1. Spectroscopic observations of SN 2016bau.

Note: All spectra were taken with the Kast spectrograph on the $3.0 \mathrm{~m}$ Shane telescope at Lick Observatory.

\begin{tabular}{cc}
\hline \hline UT Date & MJD \\
\hline $2016 / 03 / 16$ & 57463.719 \\
$2016 / 03 / 19$ & 57466.919 \\
$2016 / 04 / 03$ & 57481.671 \\
$2016 / 04 / 17$ & 57495.902 \\
$2016 / 05 / 02$ & 57510.671 \\
$2016 / 05 / 16$ & 57524.860 \\
$2016 / 05 / 30$ & 57538.741 \\
$2016 / 07 / 15$ & 57584.710 \\
\hline
\end{tabular}

Table A2. Photometry of SN 2015ap.

\begin{tabular}{|c|c|c|c|c|c|}
\hline MJD & $\begin{array}{c}B \\
(\mathrm{mag})\end{array}$ & $\begin{array}{c}V \\
(\mathrm{mag})\end{array}$ & $\begin{array}{c}R \\
(\mathrm{mag})\end{array}$ & $\begin{array}{c}I \\
(\mathrm{mag})\end{array}$ & $\begin{array}{c}\text { Telescope } \\
\text { (mag) }\end{array}$ \\
\hline 57274.4258 & $17.35 \pm 0.09$ & $17.28 \pm 0.07$ & $17.07 \pm 0.05$ & $16.75 \pm 0.06$ & KAIT \\
\hline 57275.3867 & $16.94 \pm 0.09$ & $16.83 \pm 0.06$ & $16.67 \pm 0.05$ & $16.39 \pm 0.06$ & KAIT \\
\hline 57276.3516 & $16.40 \pm 0.17$ & $16.40 \pm 0.11$ & $16.39 \pm 0.08$ & $16.05 \pm 0.07$ & KAIT \\
\hline 57279.4336 & $15.79 \pm 0.11$ & $15.84 \pm 0.06$ & $15.64 \pm 0.05$ & $15.39 \pm 0.05$ & KAIT \\
\hline 57283.4375 & $15.44 \pm 0.01$ & $15.26 \pm 0.01$ & $15.14 \pm 0.01$ & $14.93 \pm 0.01$ & Nickel \\
\hline 57285.5078 & $15.64 \pm 0.08$ & $15.44 \pm 0.04$ & $15.20 \pm 0.03$ & $14.90 \pm 0.03$ & KAIT \\
\hline 57286.4062 & $15.70 \pm 0.04$ & $15.48 \pm 0.03$ & $15.14 \pm 0.02$ & $14.87 \pm 0.02$ & KAIT \\
\hline 57287.4375 & $15.91 \pm 0.05$ & $15.47 \pm 0.03$ & $15.18 \pm 0.03$ & $14.87 \pm 0.03$ & KAIT \\
\hline 57288.4102 & $15.89 \pm 0.05$ & $15.51 \pm 0.03$ & $15.17 \pm 0.03$ & $14.86 \pm 0.03$ & KAIT \\
\hline 57290.3555 & $16.07 \pm 0.07$ & $15.55 \pm 0.05$ & $15.20 \pm 0.03$ & $14.87 \pm 0.03$ & KAIT \\
\hline 57291.3594 & $16.30 \pm 0.09$ & $15.58 \pm 0.04$ & $15.19 \pm 0.03$ & $14.88 \pm 0.013$ & KAIT \\
\hline 57297.4023 & $17.06 \pm 0.20$ & $16.27 \pm 0.07$ & $15.71 \pm 0.04$ & $15.19 \pm 0.04$ & KAIT \\
\hline 57298.3906 & $17.30 \pm 0.12$ & $16.20 \pm 0.04$ & $15.75 \pm 0.03$ & $15.23 \pm 0.03$ & KAIT \\
\hline 57299.5039 & $17.35 \pm 0.15$ & $16.53 \pm 0.05$ & $15.91 \pm 0.03$ & $15.22 \pm 0.03$ & KAIT \\
\hline 57301.4258 & $17.52 \pm 0.11$ & $16.48 \pm 0.04$ & $15.98 \pm 0.03$ & $15.35 \pm 0.03$ & KAIT \\
\hline 57302.3125 & $17.43 \pm 0.01$ & $16.37 \pm 0.01$ & $15.86 \pm 0.01$ & $15.33 \pm 0.01$ & Nickel \\
\hline 57302.3672 & $17.47 \pm 0.08$ & $16.52 \pm 0.04$ & $16.05 \pm 0.03$ & $15.46 \pm 0.03$ & KAIT \\
\hline 57304.4727 & $17.68 \pm 0.12$ & $16.76 \pm 0.07$ & $16.19 \pm 0.04$ & $15.57 \pm 0.03$ & KAIT \\
\hline 57305.3828 & $17.90 \pm 0.12$ & $16.76 \pm 0.05$ & $16.27 \pm 0.03$ & $15.61 \pm 0.03$ & KAIT \\
\hline 57306.3516 & $17.88 \pm 0.18$ & $16.84 \pm 0.06$ & $16.26 \pm 0.04$ & $15.65 \pm 0.04$ & KAIT \\
\hline 57307.3438 & $17.74 \pm 0.01$ & $16.72 \pm 0.01$ & $16.18 \pm 0.01$ & $15.63 \pm 0.01$ & Nickel \\
\hline 57307.3945 & $17.82 \pm 0.13$ & $16.86 \pm 0.04$ & $16.27 \pm 0.03$ & $15.76 \pm 0.04$ & KAIT \\
\hline 57308.3633 & $17.82 \pm 0.12$ & $16.83 \pm 0.05$ & $16.34 \pm 0.03$ & $15.75 \pm 0.04$ & KAIT \\
\hline 57309.4609 & $17.91 \pm 0.16$ & $16.89 \pm 0.08$ & $16.35 \pm 0.04$ & $15.73 \pm 0.04$ & KAIT \\
\hline 57317.3047 & $17.98 \pm 0.02$ & $17.10 \pm 0.01$ & $16.64 \pm 0.01$ & $15.99 \pm 0.01$ & Nickel \\
\hline 57317.4492 & $18.31 \pm 0.24$ & $17.25 \pm 0.08$ & $16.75 \pm 0.05$ & $16.02 \pm 0.04$ & KAIT \\
\hline 57318.3008 & $18.19 \pm 0.27$ & $17.30 \pm 0.09$ & $16.72 \pm 0.05$ & $16.12 \pm 0.05$ & KAIT \\
\hline 57319.3984 & $18.22 \pm 0.20$ & $17.48 \pm 0.12$ & $16.80 \pm 0.05$ & $16.12 \pm 0.07$ & KAIT \\
\hline 57325.4297 & $18.25 \pm 0.38$ & $17.14 \pm 0.14$ & $17.15 \pm 0.09$ & $16.19 \pm 0.07$ & KAIT \\
\hline 57326.3594 & $18.42 \pm 0.29$ & $17.43 \pm 0.07$ & $17.03 \pm 0.05$ & $16.35 \pm 0.04$ & KAIT \\
\hline 57327.4297 & $17.98 \pm 0.26$ & $17.40 \pm 0.12$ & - & - & KAIT \\
\hline 57330.3906 & $18.37 \pm 0.22$ & $17.58 \pm 0.08$ & $17.20 \pm 0.06$ & $16.46 \pm 0.05$ & KAIT \\
\hline 57331.3359 & $18.63 \pm 0.22$ & $17.72 \pm 0.08$ & $17.16 \pm 0.04$ & $16.59 \pm 0.05$ & KAIT \\
\hline 57332.3047 & $18.21 \pm 0.01$ & $17.47 \pm 0.01$ & $17.11 \pm 0.01$ & $16.47 \pm 0.01$ & Nickel \\
\hline 57332.3555 & $18.31 \pm 0.18$ & $17.57 \pm 0.07$ & $17.21 \pm 0.04$ & $16.56 \pm 0.04$ & KAIT \\
\hline 57333.3477 & $18.19 \pm 0.17$ & $17.73 \pm 0.09$ & $17.23 \pm 0.06$ & $16.56 \pm 0.05$ & KAIT \\
\hline 57334.3789 & $18.35 \pm 0.16$ & $17.66 \pm 0.08$ & $17.25 \pm 0.06$ & $16.51 \pm 0.05$ & KAIT \\
\hline 57337.3281 & $18.33 \pm 0.20$ & $18.01 \pm 0.18$ & $17.33 \pm 0.05$ & $16.85 \pm 0.06$ & KAIT \\
\hline 57338.2852 & $18.62 \pm 0.28$ & $17.79 \pm 0.12$ & $17.30 \pm 0.05$ & $16.73 \pm 0.05$ & KAIT \\
\hline 57339.3125 & $18.45 \pm 0.22$ & $17.83 \pm 0.08$ & $17.46 \pm 0.05$ & $16.75 \pm 0.06$ & KAIT \\
\hline 57339.3438 & $18.28 \pm 0.02$ & $17.64 \pm 0.01$ & $17.27 \pm 0.02$ & $16.66 \pm 0.01$ & Nickel \\
\hline 57340.3594 & $18.52 \pm 0.18$ & $17.76 \pm 0.07$ & $17.40 \pm 0.06$ & $16.73 \pm 0.06$ & KAIT \\
\hline 57343.3906 & $18.31 \pm 0.02$ & $17.66 \pm 0.01$ & $17.38 \pm 0.02$ & $16.64 \pm 0.01$ & Nickel \\
\hline 57347.3164 & $18.51 \pm 0.03$ & $17.73 \pm 0.01$ & $17.51 \pm 0.02$ & $17.02 \pm 0.02$ & Nickel \\
\hline 57361.2656 & $18.55 \pm 0.02$ & $18.14 \pm 0.01$ & $17.93 \pm 0.02$ & $17.19 \pm 0.01$ & Nickel \\
\hline 57425.1641 & $19.22 \pm 0.06$ & $19.63 \pm 0.07$ & - & - & Nickel \\
\hline 57431.1250 & $19.51 \pm 0.10$ & $19.48 \pm 0.10$ & $19.05 \pm 0.34$ & - & Nickel \\
\hline 57444.1328 & $19.67 \pm 0.09$ & $20.06 \pm 0.10$ & $19.82 \pm 0.09$ & $18.93 \pm 0.06$ & Nickel \\
\hline
\end{tabular}


22 Aryan et al.

Table A3. Log of HST observations of SN 2015ap.

Note: All exposures were $710 \mathrm{~s}$ and there were no detections.

\begin{tabular}{cc}
\hline \hline UT Date & Filter \\
& \\
\hline $2017 / 08 / 16$ & F555W \\
$2017 / 02 / 19$ & F814W \\
$2016 / 04 / 03$ & F555W \\
$2016 / 04 / 17$ & F814W \\
\hline
\end{tabular}


Table A4. Photometry of SN 2016bau

\begin{tabular}{|c|c|c|c|c|c|c|}
\hline$\overline{\mathrm{MJD}}$ & $\begin{array}{c}B \\
(\mathrm{mag})\end{array}$ & $\begin{array}{c}V \\
(\mathrm{mag})\end{array}$ & $\begin{array}{c}R \\
(\mathrm{mag})\end{array}$ & $\begin{array}{c}\text { clear } \\
\text { (mag) }\end{array}$ & $\begin{array}{c}I \\
(\mathrm{mag})\end{array}$ & Telescope \\
\hline 57462.384 & - & - & 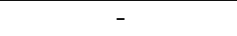 & $16.896 \pm 0.190$ & - & \\
\hline 57463.381 & $17.196 \pm 0.160$ & $16.691 \pm 0.131$ & $16.488 \pm 0.165$ & $16.553 \pm 0.226$ & $16.381 \pm 0.124$ & KAIT \\
\hline 57464.338 & $16.843 \pm 0.119$ & $16.408 \pm 0.080$ & $16.180 \pm 0.102$ & $16.199 \pm 0.103$ & $16.079 \pm 0.086$ & KAIT \\
\hline 57465.324 & $16.879 \pm 0.120$ & $16.192 \pm 0.066$ & $16.027 \pm 0.070$ & $15.967 \pm 0.131$ & $15.809 \pm 0.106$ & KAIT \\
\hline 57466.310 & $16.726 \pm 0.134$ & $16.005 \pm 0.057$ & $15.852 \pm 0.055$ & $15.838 \pm 0.105$ & $15.661 \pm 0.063$ & KAIT \\
\hline 57467.330 & $16.787 \pm 0.253$ & $15.890 \pm 0.103$ & $15.608 \pm 0.098$ & $15.767 \pm 0.156$ & $15.451 \pm 0.128$ & KAIT \\
\hline 57470.385 & $16.762 \pm 0.165$ & $15.741 \pm 0.071$ & $15.376 \pm 0.074$ & $15.374 \pm 0.061$ & $15.165 \pm 0.091$ & KAIT \\
\hline 57471.397 & $16.401 \pm 0.176$ & $15.589 \pm 0.059$ & $15.278 \pm 0.053$ & $15.288 \pm 0.062$ & $15.114 \pm 0.056$ & KAIT \\
\hline 57472.397 & $16.358 \pm 0.140$ & $15.477 \pm 0.061$ & $15.202 \pm 0.051$ & $15.254 \pm 0.065$ & $15.031 \pm 0.055$ & KAIT \\
\hline 57473.355 & $16.282 \pm 0.114$ & $15.467 \pm 0.049$ & $15.196 \pm 0.049$ & $15.206 \pm 0.072$ & $14.982 \pm 0.055$ & KAIT \\
\hline 57474.383 & $16.106 \pm 0.315$ & $15.464 \pm 0.083$ & $15.107 \pm 0.063$ & $15.154 \pm 0.075$ & $14.882 \pm 0.114$ & KAIT \\
\hline 57476.373 & $16.079 \pm 0.131$ & $15.398 \pm 0.048$ & $15.026 \pm 0.049$ & $15.067 \pm 0.079$ & $14.806 \pm 0.052$ & KAIT \\
\hline 57477.367 & $16.274 \pm 0.088$ & $15.348 \pm 0.042$ & $15.048 \pm 0.042$ & $15.073 \pm 0.046$ & $14.787 \pm 0.042$ & KAIT \\
\hline 57478.332 & $16.340 \pm 0.082$ & $15.376 \pm 0.044$ & $15.005 \pm 0.042$ & $15.055 \pm 0.080$ & $14.744 \pm 0.040$ & AIT \\
\hline 57479.395 & $16.290 \pm 0.123$ & $15.365 \pm 0.054$ & $14.989 \pm 0.053$ & $15.039 \pm 0.089$ & $14.750 \pm 0.060$ & AIT \\
\hline 57480.345 & $16.440 \pm 0.087$ & $15.422 \pm 0.034$ & $15.037 \pm 0.038$ & $15.062 \pm 0.045$ & $14.753 \pm 0.056$ & AIT \\
\hline 57481.338 & $16.487 \pm 0.207$ & $15.444 \pm 0.070$ & $15.102 \pm 0.065$ & $15.105 \pm 0.073$ & $14.713 \pm 0.062$ & AIT \\
\hline 57482.341 & $16.801 \pm 0.142$ & $15.527 \pm 0.053$ & $15.057 \pm 0.045$ & $15.132 \pm 0.065$ & $14.784 \pm 0.049$ & AIT \\
\hline 57485.358 & $17.453 \pm 0.207$ & $15.691 \pm 0.115$ & $15.237 \pm 0.093$ & $15.320 \pm 0.090$ & $15.039 \pm 0.090$ & KAIT \\
\hline 57491.352 & $17.914 \pm 0.243$ & $16.282 \pm 0.118$ & $15.730 \pm 0.119$ & $15.744 \pm 0.141$ & $15.224 \pm 0.116$ & KAIT \\
\hline 57492.352 & $17.765 \pm 0.159$ & $16.376 \pm 0.103$ & $15.776 \pm 0.085$ & $15.824 \pm 0.137$ & $15.262 \pm 0.073$ & KAIT \\
\hline 57494.257 & $17.979 \pm 0.169$ & $16.484 \pm 0.108$ & $15.896 \pm 0.111$ & $15.941 \pm 0.148$ & $15.392 \pm 0.125$ & KAIT \\
\hline 57495.299 & $18.142 \pm 0.134$ & $16.553 \pm 0.069$ & $15.914 \pm 0.071$ & $16.018 \pm 0.095$ & $15.427 \pm 0.064$ & KAIT \\
\hline 57496.272 & $18.106 \pm 0.131$ & $16.603 \pm 0.065$ & $15.970 \pm 0.063$ & $16.048 \pm 0.093$ & $15.445 \pm 0.058$ & KAIT \\
\hline 57497.268 & $18.194 \pm 0.186$ & $16.721 \pm 0.086$ & $15.966 \pm 0.065$ & $16.100 \pm 0.084$ & $15.492 \pm 0.067$ & KAIT \\
\hline 57498.330 & $18.137 \pm 0.145$ & $16.646 \pm 0.094$ & $16.065 \pm 0.087$ & $16.129 \pm 0.114$ & $15.557 \pm 0.079$ & KAIT \\
\hline 57499.317 & $18.147 \pm 0.329$ & $16.675 \pm 0.149$ & $16.111 \pm 0.141$ & $16.193 \pm 0.230$ & $15.555 \pm 0.131$ & KAIT \\
\hline 57502.338 & $18.204 \pm 0.277$ & $16.935 \pm 0.170$ & $16.180 \pm 0.204$ & $16.201 \pm 0.147$ & $15.674 \pm 0.095$ & KAIT \\
\hline 57504.271 & $18.340 \pm 0.173$ & $16.893 \pm 0.111$ & $16.291 \pm 0.114$ & $16.313 \pm 0.165$ & $15.738 \pm 0.095$ & KAIT \\
\hline 57505.327 & - & - & - & $16.297 \pm 0.148$ & - & AIT \\
\hline 57507.265 & $18.362 \pm 0.179$ & $16.974 \pm 0.109$ & $16.372 \pm 0.116$ & $16.408 \pm 0.184$ & $15.803 \pm 0.089$ & AIT \\
\hline 57508.241 & $18.348 \pm 0.248$ & $16.993 \pm 0.197$ & $16.445 \pm 0.210$ & $16.408 \pm 0.237$ & $15.837 \pm 0.163$ & AIT \\
\hline 57509.285 & $18.414 \pm 0.156$ & $17.023 \pm 0.110$ & $16.401 \pm 0.119$ & $16.441 \pm 0.181$ & $15.825 \pm 0.098$ & KAIT \\
\hline 57510.229 & - & - & - & $16.458 \pm 0.172$ & - & KAIT \\
\hline 57511.236 & $18.498 \pm 0.154$ & $17.092 \pm 0.121$ & $16.484 \pm 0.086$ & $16.447 \pm 0.178$ & $15.848 \pm 0.087$ & KAIT \\
\hline 57513.195 & $18.490 \pm 0.417$ & $17.150 \pm 0.163$ & $16.552 \pm 0.134$ & $16.517 \pm 0.195$ & $15.929 \pm 0.112$ & KAIT \\
\hline 57517.272 & $18.501 \pm 0.189$ & $17.238 \pm 0.104$ & $16.619 \pm 0.116$ & $16.594 \pm 0.221$ & $16.001 \pm 0.096$ & KAIT \\
\hline 57518.210 & $18.590 \pm 0.151$ & $17.221 \pm 0.129$ & $16.668 \pm 0.114$ & $16.591 \pm 0.139$ & $15.993 \pm 0.094$ & KAIT \\
\hline 57519.223 & $18.514 \pm 0.156$ & $17.211 \pm 0.094$ & $16.671 \pm 0.095$ & $16.612 \pm 0.127$ & $16.043 \pm 0.101$ & KAIT \\
\hline 57520.193 & $18.453 \pm 0.167$ & $17.284 \pm 0.148$ & $16.669 \pm 0.099$ & $16.651 \pm 0.349$ & $16.039 \pm 0.102$ & AIT \\
\hline 57521.203 & $18.651 \pm 0.179$ & $17.276 \pm 0.126$ & $16.737 \pm 0.121$ & $16.699 \pm 0.119$ & $16.057 \pm 0.093$ & KAIT \\
\hline 57522.186 & $18.638 \pm 0.215$ & $17.359 \pm 0.186$ & $16.744 \pm 0.190$ & $16.727 \pm 0.263$ & $16.074 \pm 0.155$ & KAIT \\
\hline 57523.259 & $18.510 \pm 0.235$ & $17.396 \pm 0.194$ & $16.697 \pm 0.149$ & $16.672 \pm 0.322$ & $16.029 \pm 0.118$ & KAIT \\
\hline 57524.206 & $18.643 \pm 0.147$ & $17.346 \pm 0.121$ & $16.717 \pm 0.116$ & $16.753 \pm 0.229$ & $16.068 \pm 0.102$ & KAIT \\
\hline 57525.193 & $18.675 \pm 0.168$ & $17.392 \pm 0.090$ & $16.802 \pm 0.118$ & $16.729 \pm 0.134$ & $16.117 \pm 0.110$ & AIT \\
\hline 57526.218 & $18.464 \pm 0.205$ & $17.358 \pm 0.120$ & $16.748 \pm 0.113$ & $16.739 \pm 0.243$ & $16.097 \pm 0.101$ & KAIT \\
\hline 57527.200 & $18.490 \pm 0.166$ & $17.401 \pm 0.092$ & $16.824 \pm 0.116$ & $16.846 \pm 0.201$ & $16.115 \pm 0.089$ & KAIT \\
\hline 57531.212 & $18.761 \pm 0.175$ & $17.450 \pm 0.096$ & $16.854 \pm 0.102$ & $16.832 \pm 0.221$ & $16.214 \pm 0.114$ & KAIT \\
\hline 57533.227 & $18.663 \pm 0.143$ & $17.541 \pm 0.109$ & $16.914 \pm 0.143$ & $16.848 \pm 0.249$ & $16.209 \pm 0.075$ & KAIT \\
\hline 57534.202 & $18.583 \pm 0.130$ & $17.519 \pm 0.085$ & $16.984 \pm 0.133$ & $16.933 \pm 0.264$ & $16.271 \pm 0.102$ & KAIT \\
\hline 57535.215 & $18.683 \pm 0.163$ & $17.558 \pm 0.110$ & $16.998 \pm 0.112$ & $16.975 \pm 0.277$ & $16.269 \pm 0.105$ & KAIT \\
\hline 57536.219 & $18.693 \pm 0.177$ & $17.587 \pm 0.101$ & $16.981 \pm 0.103$ & $16.958 \pm 0.257$ & $16.275 \pm 0.076$ & KAIT \\
\hline 57537.232 & $18.818 \pm 0.224$ & $17.562 \pm 0.098$ & $17.001 \pm 0.093$ & $17.010 \pm 0.270$ & $16.257 \pm 0.093$ & KAIT \\
\hline 57541.231 & $18.793 \pm 0.151$ & $17.682 \pm 0.086$ & $17.137 \pm 0.117$ & $16.981 \pm 0.263$ & $16.355 \pm 0.097$ & KAIT \\
\hline 57552.211 & $18.943 \pm 0.150$ & $17.819 \pm 0.124$ & $17.316 \pm 0.100$ & $17.231 \pm 0.070$ & $16.525 \pm 0.088$ & KAIT \\
\hline 57572.191 & $19.171 \pm 0.331$ & $18.126 \pm 0.132$ & $17.587 \pm \pm 0.117$ & $17.422 \pm 0.139$ & $16.842 \pm 0.118$ & KAIT \\
\hline 57574.190 & - & - & - & $17.403 \pm 0.091$ & - & KAIT \\
\hline 57576.191 & - & - & - & $17.544 \pm 0.519$ & - & KAIT \\
\hline 57471.326 & $16.267 \pm 0.022$ & $15.589 \pm 0.015$ & $15.274 \pm 0.019$ & - & $15.065 \pm 0.027$ & KAIT \\
\hline 57484.382 & $16.823 \pm 0.029$ & $15.501 \pm 0.034$ & $15.213 \pm 0.014$ & - & $14.842 \pm 0.022$ & KAIT \\
\hline 57498.264 & $17.822 \pm 0.081$ & $16.741 \pm 0.057$ & $16.069 \pm 0.031$ & - & $15.551 \pm 0.035$ & KAIT \\
\hline 57511.285 & $18.314 \pm 0.376$ & $17.054 \pm 0.046$ & $16.397 \pm 0.025$ & - & $15.843 \pm 0.616$ & KAIT \\
\hline 57535.248 & $18.563 \pm 0.058$ & $17.544 \pm 0.035$ & $16.948 \pm 0.044$ & - & $16.289 \pm 0.072$ & KAIT \\
\hline 57541.241 & $18.555 \pm 0.719$ & $17.565 \pm 0.070$ & $17.014 \pm 0.034$ & - & $16.347 \pm 0.028$ & KAIT \\
\hline 57546.216 & $18.816 \pm 0.229$ & $17.691 \pm 0.070$ & $17.139 \pm 0.028$ & - & $16.447 \pm 0.099$ & KAIT \\
\hline $0 \overline{0} \overline{5} 87.185$ & $2 \mathrm{2} 99.431 \pm 0.701$ & $18.453 \pm 0.309$ & 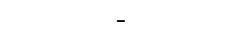 & 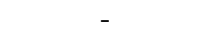 & - & KAIT \\
\hline
\end{tabular}

